\title{
Relieving tensions related to the lensing of the cosmic microwave background temperature power spectra
}

\author{
F. Couchot, S. Henrot-Versillé, O. Perdereau, S. Plaszczynski, B. Rouillé d'Orfeuil, M. Spinelli, and M. Tristram
}

\author{
Laboratoire de l'Accélérateur Linéaire, Univ. Paris-Sud, CNRS/IN2P3, Université Paris-Saclay, 91405 Orsay, France \\ e-mail: plaszczy@lal.in2p3.fr
}

Received 13 November 2015 / Accepted 24 September 2016

\begin{abstract}
The angular power spectra of the cosmic microwave background (CMB) temperature anisotropies reconstructed from Planck data seem to present "too much" gravitational lensing distortion. This is quantified by the control parameter $A_{\mathrm{L}}$ that should be compatible with unity for a standard cosmology. With the class Boltzmann solver and the profile-likelihood method, for this parameter we measure a $2.6 \sigma$ shift from 1 using the Planck public likelihoods. We show that, owing to strong correlations with the reionization optical depth $\tau$ and the primordial perturbation amplitude $A_{\mathrm{s}}$, a $\sim 2 \sigma$ tension on $\tau$ also appears between the results obtained with the low ( $\ell \leq 30$ ) and high $(30<\ell \lessgtr 2500)$ multipoles likelihoods. With Hillipop, another high- $\ell$ likelihood built from Planck data, this difference is lowered to $1.3 \sigma$. In this case, the $A_{\mathrm{L}}$ value is still in disagreement with unity by $2.2 \sigma$, suggesting a non-trivial effect of the correlations between cosmological and nuisance parameters. To better constrain the nuisance foregrounds parameters, we include the very-high- $\ell$ measurements of the Atacama Cosmology Telescope (ACT) and South Pole Telescope (SPT) experiments and obtain $A_{\mathrm{L}}=1.03 \pm 0.08$. The Hillipop+ACT+SPT likelihood estimate of the optical depth is $\tau=0.052 \pm 0.035$, which is now fully compatible with the low- $\ell$ likelihood determination. After showing the robustness of our results with various combinations, we investigate the reasons for this improvement that results from a better determination of the whole set of foregrounds parameters. We finally provide estimates of the $\Lambda$ cold dark matter parameters with our combined CMB data likelihood.
\end{abstract}

Key words. cosmic background radiation - cosmological parameters - cosmology: theory - methods: statistical cosmology: observations

\section{Introduction}

The $A_{\mathrm{L}}$ control parameter attempts to measure the degree of lensing of the cosmic microwave background (CMB) power spectra. From a set of cosmological parameters $(\Omega)$, a Boltzmann solver, such as class (Blas et al. 2011) or camb (Lewis et al. 2000), computes the angular power spectra of the temperature/polarization anisotropies $C_{\ell}(\Omega)$ and of the CMB lensing potential $C_{\ell}^{\Phi}(\Omega)$. The latter is then used to compute the distortion of the CMB spectra by the gravitational lensing (Blanchard \& Schneider 1987), which redistributes the power across multipoles while preserving the brightness in a non-trivial way (e.g. Lewis \& Challinor 2006): $\left\{C_{\ell}(\Omega), C_{\ell}^{\Phi}(\Omega)\right\} \rightarrow \tilde{C}_{\ell}(\Omega)$. As originally proposed in Calabrese et al. (2008), a phenomenological parameter, $A_{\mathrm{L}}$, that re-scales the lensing potential, is introduced. This modifies the standard scheme into: $\left\{C_{\ell}(\Omega), A_{\mathrm{L}}\right.$. $\left.C_{\ell}^{\Phi}(\Omega)\right\} \rightarrow \tilde{C}_{\ell}\left(\Omega, A_{\mathrm{L}}\right)$. Sampling the likelihood, with this parameter left free, gives access to two interesting pieces of information:

1. from the $A_{\mathrm{L}}$ posterior distribution, one can check the consistency of the data with the model; it should be compatible with 1.0 for a standard cosmology.

2 . by marginalizing over $A_{\mathrm{L}}$, one can study the impact of neglecting (to first-order) the lensing information contained in the CMB spectra.

Since its first release, the Planck Collaboration reports a value of the $A_{\mathrm{L}}$ parameter that is discrepant with one by more than $2 \sigma$. The full-mission result, based on both a high and low- $\ell$ likelihood
(Planck Collaboration XIII 2016, hereafter PCP15), is

$$
A_{\mathrm{L}}=1.22 \pm 0.10 \quad(\text { Planck TT }+ \text { lowP })
$$

(all quoted errors are 68\% CL intervals). As shown later, a profile likelihood analysis, as the one in Planck Collaboration Int. XVI (2014), rather points to a $2.6 \sigma$ discrepancy.

This "tension" may indicate a problem either on the model or the data side. The only solution for the model is to modify the computation of the geodesic deflection, i.e. to modify standard GR (Hu \& Raveri 2015; Di Valentino et al. 2016). For the data, since Planck maps undergo a complicated treatment (for an overview see Planck Collaboration I 2016), one cannot exclude small residual systematic effects that could impact the details of the likelihood function in a different way from one implementation to the other.

The anomalously high $A_{\mathrm{L}}$ value directly affects the measurement of two $\Lambda$ cold dark matter $(\Lambda \mathrm{CDM})$ parameters, the reionization optical depth $\tau$ and the primordial scalar perturbations amplitude $A_{\mathrm{s}}$. Indeed, in the high $\ell$ regime, only the $\mathcal{A}_{T} \equiv A_{\mathrm{s}} \mathrm{e}^{-2 \tau}$ combination is constrained by the temperature power spectra amplitude. However this degeneracy is broken by the lensing distortion of the CMB anisotropies since $C_{\ell}^{\Phi} \propto A_{\mathrm{s}}$ (more perturbations induce more lensing) so that both $A_{\mathrm{s}}$ and $\tau$ finally get constrained.

The aim of this work is twofold. First, to clarify the connection between the $A_{\mathrm{L}}$ tension with unity and the one that also appears on $\tau$ between the Planck public high and low- $\ell$ likelihoods, and also to show that this effect may be related to the details of the nuisance parametrization in the likelihoods. By 
using Hillipop, a high- $\ell$ likelihood that is built from Planck data, and better constraining the astrophysical foregrounds and the high- $\ell$ part of the CMB spectrum with the high-angular resolution data from Atacama Cosmology Telescope (ACT) and South Pole Telescope (SPT), we show that one can obtain a more self-consistent picture of the $\Lambda \mathrm{CDM}$ parameters. Section 2 provides an in-depth discussion about $A_{\mathrm{L}}$ using the Planck baseline likelihoods, Plik and lowTEB, and makes the link with the determination of $\tau$ explicit. Section 3 then recalls the main differences between Plik and Hillipop and discusses the first results with the latter. Then Sect. 4 describes how the inclusion of the ACT and SPT data was performed and, after various checks, discusses how their inclusion impacts the Hillipop results. Finally Sect. 5 discusses the results on the $\Lambda \mathrm{CDM}$ parameters using Hillipop in combination with other likelihoods.

\section{The Planck $A_{L}$ tension (and related parameters)}

\subsection{Planck likelihoods}

Planck's baseline uses two different likelihood codes addressing different multipole ranges (see Planck Collaboration XI 2016, hereafter Like15):

1. the high- $\ell$ likelihood (Plik) is a Gaussian likelihood that acts in the multipole range $\ell \in[30,2500]$. Data consist of a collection of angular power spectra that are derived from crosscorrelated Planck 100,143, and $217 \mathrm{GHz}$ high frequency maps. For the results in this paper, we only use the temperature likelihood.

2. the low- $\ell$ likelihood (lowTEB) is a pixel-based likelihood that essentially relyies on the Planck low frequency instrument $70 \mathrm{GHz}$ maps for polarization and on a component-separated map using all Planck frequencies for temperature. It acts in the $\ell \in[2,29]$ range.

In the Like15 terminology, "Planck TT" refers to the combination of both the high and the low- $\ell$ temperature likelihoods. In this case, only the TT component of lowTEB is used. The "Planck TT+lowP" notation combines Plik to the full lowTEB likelihood, which we label explicitly in this paper as Plik+lowTEB.

In the following, we will make use of the publicly available Plik likelihood code (plik_dx11dr2_HM_v18_TT.clik) ${ }^{1}$ with the Gaussian priors on nuisance parameters suggested by the Planck Collaboration ${ }^{2}$, where the information on foregrounds from the ACT and SPT data is propagated by a single SZ prior (PCP15, Sect. 2.3.1).

\subsection{Boltzmann solver}

The results derived in this paper make use of the Boltzmann equations solver class ${ }^{3}$ while Planck's published results were derived using $\mathrm{camb}^{4}$. Both softwares have been compared previously (Lesgourgues 2011) and have produced spectra in excellent agreement when using their respective high precision settings. More recentl, it was noticed in PCP15 that sampling from any of them gives very compatible results on $\Lambda \mathrm{CDM}$ cosmological parameters. The precise estimate of the $A_{\mathrm{L}}$ parameter is more

\footnotetext{
1 Available in the Planck Legacy Archive (PLA):

http://www. cosmos. esa.int/web/planck/pla

2 http://wiki.cosmos.esa.int/planckpla2015

3 http://class-code.net

4 http://camb.info
}

challenging since one is dealing with sub-percent effects on the spectra and thia requires extra care about differences between both softwares.

For this purpose, we sampled the $\mathrm{Plik}+$ lowTEB likelihoods in the $\Lambda \mathrm{CDM}+A_{\mathrm{L}}$ model using class v2.3.2 and obtain results almost identical to the published ones. In particular we measure

$A_{\mathrm{L}}=1.24 \pm 0.10 \quad(\mathrm{Plik}+$ lowTEB, class/MCMC $)$.

The tiny difference with respect to Eq. (1) can be traced down to a $O\left(1(\mu \mathrm{K})^{2}\right)$ difference on the high- $\ell$ part of the TT spectra (see Appendix A), but we consider that this general agreement is sufficient to perform reliable estimations. All further results will be derived consistently using class.

\subsection{Profile likelihoods}

In this paper, we also often make use of a statistical methodology based on profile-likelihoods for reasons that will be clearer in Sect. 2.5. For a given parameter $\theta$, we perform several multidimensional minimizations of the $\chi^{2} \equiv-2 \ln \mathscr{L}$ function. Each time $\theta$ is fixed to a given $\theta(i)$ value, a minimization is performed with respect to all the other parameters, and the $\chi_{\min }^{2}(i)$ value is kept. The curve interpolated through the $\left\{\theta(i), \chi_{\min }^{2}(i)\right\}$ points and offset to 0 , is known as the $\theta$ profile-likelihood: $\Delta \chi^{2}(\theta)$. We note that from the very construction procedure, the solution at the minimum of the profile always coincides with the complete best-fit solution, i.e the maximum likelihood estimate (MLE) of all the parameters. A genuine $68 \% \mathrm{CL}$ interval is obtained by thresholding the profile at one even in non-Gaussian cases (e.g., James 2007).

This statistical method, an alternative to Monte-Carlo Markov chain (MCMC) sampling, was discussed in Planck Collaboration Int. XVI (2014) and also used in Like15. Building a smooth profile from Planck data is computationally challenging since this approach requires an extreme precision on the $\chi_{\min }^{2}$ solution, typically better than 0.1 for values around $10^{4}$. This goal can be achieved using the Minuit software ${ }^{5}$ together with an increase of the class precision parameters. For the analysis presented in this paper, we have further refined the procedure described in Planck Collaboration Int. XVI (2014), as explained in Appendix B.

This procedure leads to a so-called confidence interval (e.g., James 2007). In the frequentist approach, this represents a statement on the data: when repeating the experiment many times, the probability for the reconstructed interval to cover the true value is $68 \%$. The Bayesian approach, as implemented through a MCMC method, leads to what is generally referred to as a "credible interval" derived from the probability density function of the true value. In most cases (in particular Gaussian) both intervals are very similar. However, in some cases (typically socalled banana-shaped 2D posteriors), these intervals may differ significantly (Porter 1996). In this case, the mode (or mean) of the posterior distribution does not necessarily match the best fit solution and the existence of a difference between both values indicates what is referred to as likelihood volume effects. In this paper, we will mainly focus on the details of the region around the maximum likelihood and $\mathrm{x}$ will thus use the profile-likelihood method consistently.

\footnotetext{
5 http://seal.web.cern.ch/seal/work-packages/mathlibs/ minuit/index.html
} 


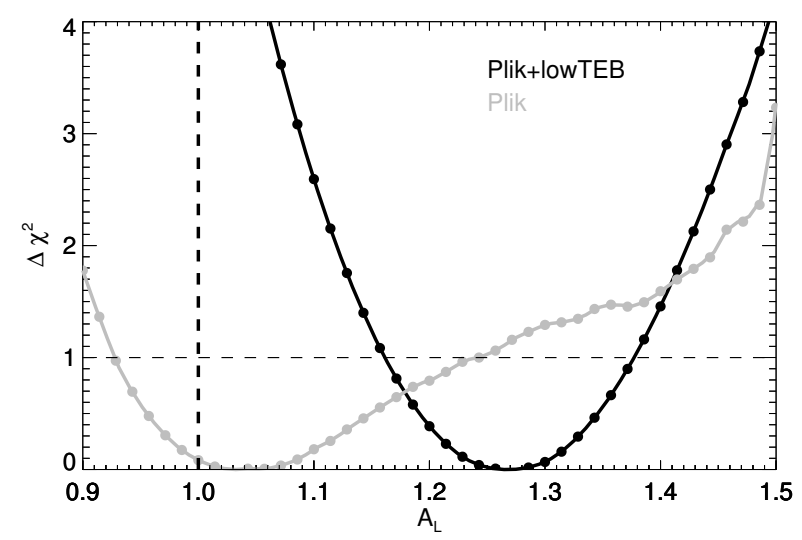

Fig. 1. Profile-likelihoods of the $A_{\mathrm{L}}$ parameter reconstructed from the Plik high $\ell$ likelihood alone (in grey) and when adding the lowTEB one (in black). The vertical dashed line recalls the expected $\Lambda$ CDM value.

\section{4. $A_{L}$ revisited}

We first build the profile-likelihood for $A_{\mathrm{L}}$ (Fig. 1) and measure $A_{\mathrm{L}}=1.26_{-0.10}^{+0.11} \quad(\mathrm{Plik}+$ lowTEB, class/profile $)$.

The shift with respect to Eq. (2) quantifies the size of the volume effects in the MCMC projection. It is of the same order of magnitude as the camb $\rightarrow$ class transition seen in Sect. 2.2. Using high-precision settings, we therefore find $A_{\mathrm{L}}$ at $2.6 \sigma$ from 1.0.

Figure 1 also shows that the $\mathrm{Plik}$-alone likelihood (in grey) gives

$A_{\mathrm{L}}=1.04_{-0.10}^{+0.20} \quad(\mathrm{Plik}, \mathrm{cl}$ ass/profile $)$,

which is compatible with 1.0. This difference from the Planck baseline result (Eq. (3)) seems to come from a tension between the low and high- $\ell$ likelihoods. Moreover, using a prior of the kind $\tau=0.07 \pm 0.02$ (as in Like15) leads to $A_{\mathrm{L}}=1.16 \pm 0.09$, which goes in the same direction as Plik+lowTEB. This connection with $\tau$ will be discussed in the following section.

\subsection{High-vs. low- $\ell$ likelihood results on $\tau$ and $A_{S}$}

We further investigate the high vs. low- $\ell$ likelihood tensions from the point of view of two other parameters that are strongly correlated to $A_{\mathrm{L}}$ : the reionization optical depth $\tau$ and the scalar perturbation amplitude $A_{\mathrm{s}}$.

Figure 2 shows, in black, the $\tau$ profile-likelihood reconstructed with Plik only, which gives

$$
\tau=0.172_{-0.042}^{+0.038} \quad \text { (Plik). }
$$

This is higher than the maximum of the posterior reported in Like15 (Fig. 45) that is around 0.14 and is partly due to a volume effect (Sect. 2.3) and partly because of the class/camb difference highlighted in Appendix A. Using consistently class and the same methodology, our result is $2.2 \sigma$ away from the $\tau$ determination with the lowTEB likelihood for which the profile-likelihood gives the same results as the MCMC marginalization (Like15):

$$
\tau=0.067_{-0.021}^{+0.023} \quad \text { (lowTEB), }
$$

represented as a blue line on Fig. 2.

Without fixing $A_{\mathrm{L}}$ to 1 (grey curve on Fig. 2) the constraint on $\tau$ is much weaker, which illustrates the fact that the lensing of the CMB anisotropies in the high- $\ell$ likelihood is the main contributor to the $\tau$ measurement. Some constraining power still

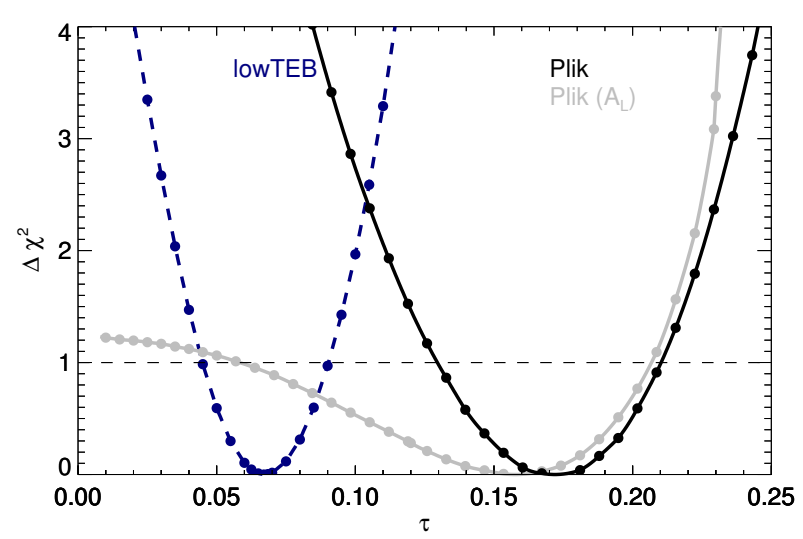

Fig. 2. High vs. low- $\ell$ Planck likelihood constraints on $\tau$. The high- $\ell$ result is obtained with Plik (only) and is shown in black while the low- $\ell$ one is in dashed blue. Both are obtained within the $\Lambda$ CDM model. The grey profile shows the result for Plik when $A_{\mathrm{L}}$ is left free in the fits.

remains in particular for large $\tau$ values: this is due to the fact that the degeneracy between $A_{\mathrm{s}}$ and $\tau$ is broken for large $\tau$ when the reionisation bump at low $\ell$ enters the multipole range of Plik $(\ell>30$; Hu \& White see 1997).

The discrepancy highlighted in Fig. 2 is directly related to the $A_{\mathrm{L}}$ problem (Fig. 1), but is simpler to study. The high- $\ell$ likelihood requires a large $\tau$ value that is in tension with the low- $\ell$-based result. In the $A_{\mathrm{L}}$ test (Fig. 1) one combines both likelihoods.lowTEB pulls $\tau$ down. To match the spectra amplitude $\left(\mathcal{A}_{T}\right)$, the high- $\ell$ likelihood pulls $A_{\mathrm{s}}$ down. Then $A_{\mathrm{L}}$, being fully anti-correlated to $A_{\mathrm{s}}$ ( since $C_{\ell}^{\Phi} \propto A_{\mathrm{L}} A_{\mathrm{s}}$ ), shifts to adjust the lensed model to the data again.

Because of the $\mathcal{A}_{T}$ degeneracy, the Plik-only estimate of $A_{\mathrm{s}}$ is also expected to be high. Indeed from a similar profilelikelihood analysis we obtain

$\ln \left(10^{10} A_{\mathrm{s}}\right)=3.270_{-0.078}^{+0.058} \quad(\mathrm{Plik})$,

again discrepant by more than $2 \sigma$ with the results from Plik+lowTEB, $3.089 \pm 0.036(\mathrm{PCP} 15)$.

In summary, the Plik high- $\ell$ likelihood alone converges to a consistent solution, $A_{\mathrm{L}} \simeq 1$, but with large $\tau$ and $A_{\mathrm{S}}$ values. Constraining $\tau$ down by adding the lowTEB likelihood (or a low prior) is compensated in the fits by increasing $A_{\mathrm{L}}$ to match the data. To investigate the stability of those results, we will now use another Planck high- $\ell$ likelihoods.

\section{The Hillipop likelihood}

\subsection{Description}

Hillipop is one of the Planck high- $\ell$ likelihoods developed for the 2015 data release and is shorty described in Like15. Similarly to Plik, it is a Gaussian likelihood based on cross-spectra from the HFI 100, 143, and $217 \mathrm{GHz}$ maps. The estimate of crossspectra on data is performed using Xpol, a generalization of the Xspec algorithm (Tristram et al. 2005) to polarization. Figure 3 shows the combined TT spectrum with respect to the best-fit model that will be deepened later on.

The differences with Plik were mentioned in Like15. The most significant are:

- we use all the 15 half-mission cross-spectra built from the 100,143 , and $217 \mathrm{GHz}$ maps while Plik uses only five of them; 


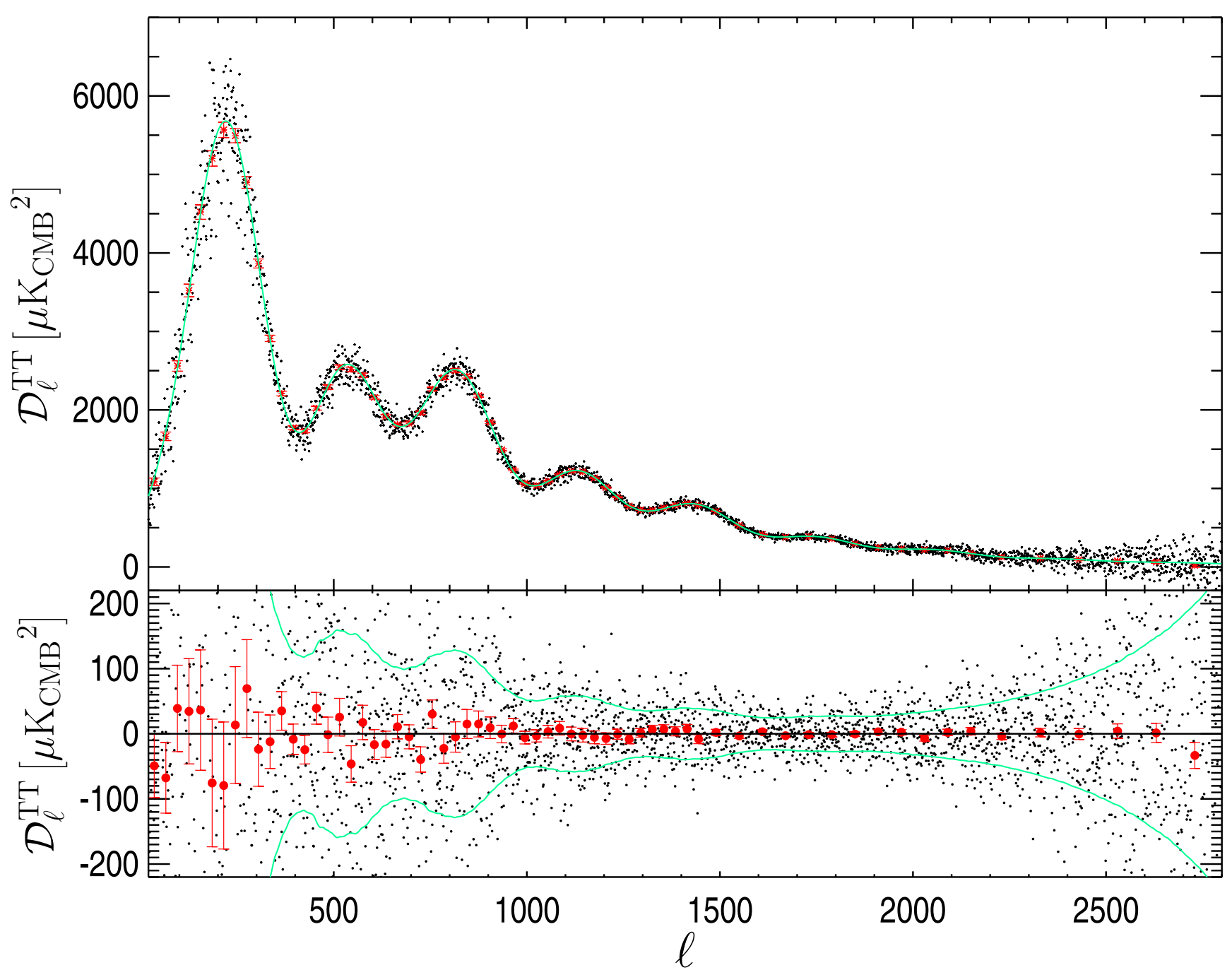

Fig. 3. Hillipop foreground-subtracted combined power-spectrum $\left(\mathcal{D}_{\ell}=\ell(\ell+1) C_{\ell} / 2 \pi\right)$ at each multipole (black points) and binned (red points) with respect to the best-fit model. The bottom plot shows the residuals. The green line shows the standard deviation as estimated from the covariance matrix.

- we apply inter-calibration coefficients at the map level, resulting in five free parameters (one is fixed) while Plik uses two at the spectrum level;

- we use point-sources masks that were obtained from a refined procedure that extracts Galactic compact structures;

- as a result, our galactic dust component follows closely and is parametrized by the power law discussed in Planck Collaboration Int. XXX (2016);

- we use foreground templates derived from Planck Collaboration XXX (2014) for the cosmic infrared background (CIB), and Planck Collaboration XXII (2016) for the SZ;

- we use all multipole values (i.e., do not bin the spectra).

Using Hillipop leads to $\Lambda \mathrm{CDM}$ estimates that are very compatible with the other Planck ones but on $A_{\mathrm{s}}$ and $\tau$ (Like15, Sect. 4.2). Using a prior on $\tau$ of $0.07 \pm 0.02$, we obtain with Hillipop $\tau=0.075 \pm 0.019$, while Plik gives a higher value $\tau=0.085 \pm 0.018$ (Like15). Given the relation between $\tau$ and $A_{\mathrm{L}}$ discussed in Sect. 2.5, we can therefore expect different results on $A_{\mathrm{L}}$.

\subsection{Results}

The profile-likelihoods of $A_{\mathrm{L}}$ derived from Hillipop with and without lowTEB is shown in Fig. 4. The Hillipop-alone profile is minimum near $A_{\mathrm{L}}=1.30$ but is very broad : a $68 \% \mathrm{CL}$ interval goes from .96 up to 1.42 . We therefore conclude that Hillipop alone does not give a strict constraint on $A_{\mathrm{L}}$. In combination with lowTEB, using the same procedure as described in Sect. 2.4, we obtain

$A_{\mathrm{L}}=1.22_{-0.10}^{+0.11} \quad$ (Hillipop+lowTEB).

This is slightly lower than the result obtained with Plik (Eq. (3)) but still discrepant with one by about $2 \sigma$.

Within the $\Lambda$ CDM model, Fig. 5 compares Hillipop vs. lowTEB results on $\tau$. The Hillipop profile on $\tau$ gives

$\tau=0.134_{-0.048}^{+0.038} \quad$ (Hillipop).

This is lower than the Plik result with similar error bars (Eq. (5)) and lies within $1.3 \sigma$ of the low- $\ell$ measurement (Eq. (6)). In the $\Lambda \mathrm{CDM}+A_{\mathrm{L}}$ case, Hillipop only gives an upper limit. The difference with Plik $\tau$ profile (Fig. 2) is the sign of different correlations between $A_{\mathrm{L}}$ and $\tau$ in these likelihoods. 
F. Couchot et al.: Relieving tensions related to $A_{\mathrm{L}}$

Table 1. Nuisance parameters for the Hillipop likelihood and Gaussian prior used during the likelihood maximization.

\begin{tabular}{llc}
\hline \hline Name & Definition & Prior (if any) \\
\hline \multicolumn{3}{c}{ Instrumental } \\
\hline$c_{0}$ & map calibration (100-hm1) & $1.000 \pm 0.002$ \\
$c_{1}$ & map calibration (100-hm2) & $1.000 \pm 0.002$ \\
$c_{2}$ & map calibration (143-hm1) & fixed to 1. \\
$c_{3}$ & map calibration (143-hm2) & $1.0000 \pm 0.002$ \\
$c_{4}$ & map calibration (217-hm1) & $1.0025 \pm 0.002$ \\
$c_{5}$ & map calibration (217-hm2) & $1.0025 \pm 0.002$ \\
$A$ & absolute calibration & $1.0000 \pm 0.0025$ \\
\hline
\end{tabular}

\begin{tabular}{ll}
\multicolumn{3}{c}{ Foreground modelling } \\
\hline$A_{\mathrm{PS}}^{100 \times 100}$ & PS amplitude in TT $(100 \times 100 \mathrm{GHz})$ \\
$A_{\mathrm{PS}}^{100143}$ & PS amplitude in TT $(100 \times 143 \mathrm{GHz})$ \\
$A_{\mathrm{PS}}^{100 \times 217}$ & PS amplitude in TT $(100 \times 217 \mathrm{GHz})$ \\
$A_{\mathrm{PS}}^{143 \times 143}$ & PS amplitude in TT $(143 \times 143 \mathrm{GHz})$ \\
$A_{\mathrm{PS}}^{143 \times 217}$ & PS amplitude in TT $(143 \times 217 \mathrm{GHz})$ \\
$A_{\mathrm{PS}}^{217 \times 217}$ & PS amplitude in TT $(217 \times 217 \mathrm{GHz})$ \\
$A_{\mathrm{SZ}}$ & scaling for the tSZ template \\
$A_{\mathrm{CIB}}$ & scaling for the CIB template \\
$A_{\mathrm{kSZ}}$ & scaling for the kSZ template \\
$A_{\mathrm{SZ} \times \mathrm{CIB}}$ & scaling parameter for the \\
& cross-correlation between kSZ and CIB \\
$A_{\mathrm{dust}}^{\mathrm{TT}}$ & scaling parameter for the dust in TT \\
\hline
\end{tabular}

Notes. The calibration factors are taken from Planck Collaboration VIII (2016) and the amplitude of the dust template extrapolated from the $353 \mathrm{GHz}$ (Planck Collaboration XXX 2014). A prior on $A_{\mathrm{CIB}}$ is applied to relieve some degree of degeneracy among foregrounds when $\mathrm{ACT}+\mathrm{SPT}$ data are not used. We have put in boldface the parameters that are common to the ACT and SPT likelihoods in the combined fit (see Sect. 4).

One of the difference between the two likelihoods is in the definition of the foreground models. Moreover, with Planck data only, the accuracy on the foreground parameters is weak (especially for SZ and CIB amplitudes). In the next section, we will use the very-high- $\ell$ datasets. This both adds constraints on lensing through the high multipoles and better determines the foregrounds parameters and possibly modifies the non-trivial correlations between nuisance and cosmological parameters.

\section{Adding very-high- $\ell$ data to constrain the foregrounds}

\subsection{Datasets}

Atacama Cosmology Telescope. We use the final ACT temperature power spectra presented in Das et al. (2014). These are $148 \times 148,148 \times 218$, and $218 \times 218$ power spectra built from observations performed on two different sky areas (south and equatorial) and during several seasons, for multipoles between 1000 and 10000 (for $148 \times 148$ ), and 1500 to 10000 otherwise.

South Pole Telescope. We use two distinct datasets from SPT.

The higher $\ell$ part, dubbed SPT_high, uses results, described in Reichardt et al. (2012), from observations at 95, 150, and

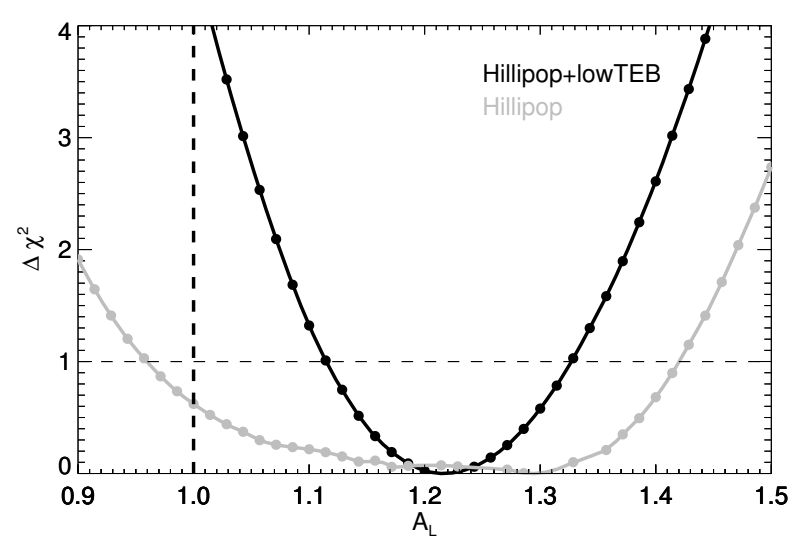

Fig. 4. Profile-likelihoods of the $A_{\mathrm{L}}$ parameter reconstructed from the Hillipop likelihood alone (in grey) and when adding lowTEB (in black). The vertical dashed line recalls the expected $\Lambda C D M$ value.

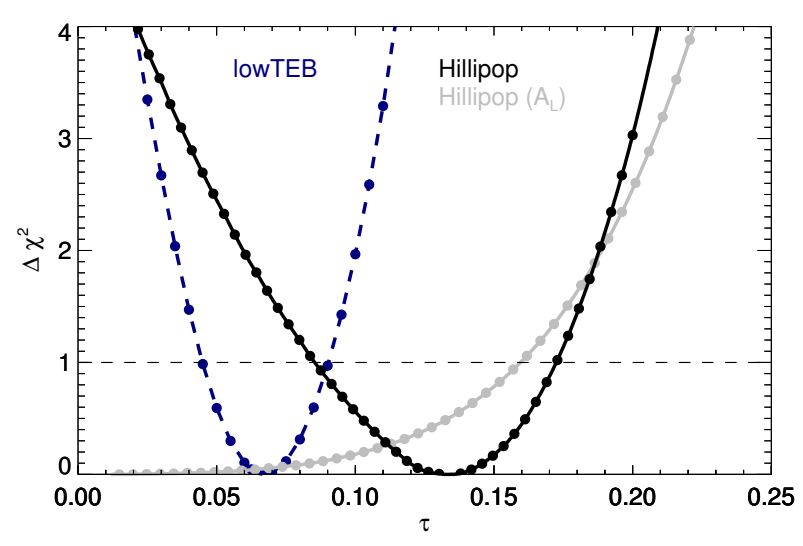

Fig. 5. Profile-likelihoods of the $\tau$ parameter using only the high- $\ell$ Hillipop likelihood: $\Lambda \mathrm{CDM}+A_{\mathrm{L}}$ free in the fits (in grey) and $\Lambda \mathrm{CDM}$ with fixed $A_{\mathrm{L}}=1$ (in black).

$220 \mathrm{GHz}$ from the SPT-SZ survey. Their cross-spectra cover the $\ell$ range between 2000 and 10000 . These measurements were calibrated using WMAP 7yr data. A more recent analysis from the complete $\sim 2500 \mathrm{deg}^{2}$ area of the SPT-SZ survey is presented in George et al. (2015), dubbed SPT_high2014 hereafter. In this later release, cross spectra cover a somewhat broader $\ell$ range, between 2000 and 13000 . Both sets of cross spectra are however quite similar, but the later comes with a covariance matrix that includes calibration uncertainties. Its use makes our work harder since it was calibrated on the Planck 2013 data, which in turn had a calibration offset of $1 \%$ (at the map level) with respect to the Planck 2015 spectra. We thus prefer to use Reichardt et al. (2012) dataset as a baseline in our analyses, with free calibration parameters to match other datasets. We have checked that all results presented in this paper are stable when switching to George et al. (2015), in which case we have to set strict priors on recalibration parameters owing to the form of the associated covariance matrix.

We also include the Story et al. (2013) dataset, dubbed SPT_low, consisting of a $150 \mathrm{GHz}$ power spectrum which ranges from $\ell=650$ to 3000 . Some concerns were raised in Planck Collaboration XVI (2014) about the compatibility of this dataset with Planck data. The tension was actually traced to be with the WMAP+SPT cosmology and the Planck and SPT_low power spectra were found to be broadly consistent with each other (Planck Collaboration XVI 2014). As will be shown later, we do 
Table 2. Conversion factors used for the foreground template extrapolation to ACT and SPT bandpasses with the CIB SED.

\begin{tabular}{lcccc}
\hline \hline Dataset & $\begin{array}{c}\text { Channel } \\
(\mathrm{GHz})\end{array}$ & $\mathrm{MJy} \mathrm{sr}^{-1} / \mathrm{K}_{\mathrm{CMB}}$ & $\begin{array}{c}\text { HFI freq. } \\
(\mathrm{GHz})\end{array}$ & Conversion \\
\hline ACT & 148 & 401.936 & 143 & 0.85 \\
& 218 & 485.311 & 217 & 1.056 \\
\hline SPT & 95 & 234.042 & 100 & 1.090 \\
& 150 & 413.540 & 143 & 0.7688 \\
& 220 & 477.017 & 217 & 1.061 \\
\hline
\end{tabular}

not see any sign of tension between the Planck 2015 data and the SPT_low dataset, nor any reason to exclude it.

\subsection{Foregrounds modelling}

For the very-high- $\ell$ astrophysical foregrounds, we chose to use a model as coherent as possible with what has been set-up for Hillipop, i.e., the same templates for tSZ, kSZ, CIB, and $\mathrm{tSZ} \times \mathrm{CIB}$. Since they have been computed for the Planck frequencies and bandpasses, we have to extrapolate them to the ACT and SPT respective effective frequencies and bandpasses. For tSZ, we scale the template with the usual $f_{v}=x \operatorname{coth} x / 2-4$ function (where $x=h v / k_{\mathrm{B}} T_{\mathrm{CMB}}$ ), using the effective frequencies for the SZ spectral distribution given in Dunkley et al. (2013). For CIB and $\mathrm{tSZ} \times \mathrm{CIB}$, we start from templates in $\mathrm{Jy}^{2} \mathrm{Sr}^{-1}$ in the IRAS convention $(v I(v)=$ cste spectrum $)$ for Planck effective frequencies and bandpasses. For CIB, we use the conversion factors from Planck to the ACT/SPT effective frequencies and bandpasses, assuming the Béthermin et al. (2012) SED for the CIB combined with unit conversion factors to $K_{\mathrm{CMB}}$, for the ACT and SPT bandpasses (Planck Collaboration IX 2014). These factors are given in Table 2.

For the tSZ $\times$ CIB component of the $\left(v_{1} \times v_{2}\right)$ cross-spectrum (from the ACT or SPT dataset), we scale the nearest HFI crossspectrum $\left(v_{1}^{P} \times v_{2}^{P}\right)$ using the ratio

$S_{v_{1}, v_{2}}=\frac{f_{v_{1}} C_{v_{2}}+f_{v_{2}} C_{v_{1}}}{f_{v_{1}^{P}} C_{v_{2}^{P}}+f_{v_{2}^{P}} C_{v_{1}^{P}}}$

and then convert it to $K_{\mathrm{CMB}}$ using the factors computed from the for the ACT and SPT bandpasses, as above. This scaling applies at the $15 \%$ level for the HFI cross-frequency templates, choosing the $143 \times 143$ one as a reference.

In addition, a few more specific templates have been added to each datasets:

- Point sources: to mask resolved point sources, ACT and SPT used their own settings to match each instrument's sensitivity and angular resolution. The unresolved point source populations in each case are thus different, so we introduce extra nuisance parameters to model them. We model the unresolved point source components in the ACT and SPT spectra with one amplitude $A_{\mathrm{PS}}^{v_{1} \times v_{2}}$ parameter per cross-spectrum. Consequently, this introduces six nuisance parameters for the ACT, six for the SPT_high, and one for the SPT_low datasets, respectively (see Table 3 ).

- Galactic dust: following Dunkley et al. (2013) and Das et al. (2014), we model the dust contribution in the ACT power spectra as a power law

$$
\mathcal{D}_{\ell}^{\text {dust }}(i, j)=A_{\text {dust }}^{\mathrm{ACT}}\left(\frac{\ell}{3000}\right)^{-0.7}\left(\frac{v_{i} v_{j}}{v_{0}^{2}}\right)^{3.8}\left[\frac{g\left(v_{i}\right) g\left(v_{j}\right)}{g\left(v_{0}\right)^{2}}\right] .
$$

Table 3. Summary of the characteristics of the very-high- $\ell$ data used in this analysis.

\begin{tabular}{cccc}
\hline \hline Dataset & Freq. (GHz) & \#Spectra & \#Nuisances \\
\hline SPT_low ......... & 150 & 1 & 2 \\
SPT_high ........ & $95,150,220$ & 6 & 9 \\
ACT south/equat ... & 148,218 & 6 & 12 \\
\hline
\end{tabular}

Notes. Each experiment's likelihood includes map calibrations and residual point source levels, which result in a number of additional nuisance parameters shown in the last column. In combined fits, the SZ and CIB foreground templates are common with Hillipop.

We therefore introduce two nuisance parameters, one for each part of the ACT dataset, and set the reference frequency $v_{0}$ at $150 \mathrm{GHz}$.

For the SPT datasets, following Reichardt et al. (2012), we use a fixed template, with amplitudes $0.16,0.21$, and $2.19 \mu K_{\mathrm{CMB}}^{2}$ at 95,150 , and $218 \mathrm{GHz}$, respectively and an $\ell^{-1.2}$ spatial dependency.

\subsection{Likelihoods}

We compute one likelihood for each of the five very-high- $\ell$ datasets following the method described in Dunkley et al. (2013). We use the respective published window functions to bin the (CMB + foregrounds) model, and the released covariance matrices to compute the likelihood. In all cases, these include beam uncertainties. Since we combine different datasets, we introduced nine additional nuisance parameters to account for their relative calibration uncertainties (at map level). Figure 6 shows a comparison of all the foreground-subtracted CMB spectra for the $150 \times 150$ component (which is almost common to all experiments), and Table 3 summarizes the characteristics of the datasets we use in the VHL likelihoods.

A detailed inspection of all cross-spectra per frequency and component has been performed and does not reveal any inconsistency with the Planck data. More details are given in Appendix C.

\subsection{First results and global consistency check}

We first check that the combination of the ACT+SPT likelihoods (hereafter VHL) gives results consistent with Hillipop. We therefore sample the Hillipop and VHL likelihoods independently and compare their $\Lambda$ CDM estimates in Fig. 7. Both datasets lead to similar cosmological parameters. However, an accurate measurement of $\Omega_{\mathrm{b}} h^{2}$ and $\Omega_{\mathrm{c}} h^{2}$ requires a precise determination of the relative amplitudes of the CMB acoustic peaks for which the very-high- $\ell$ datasets are less sensitive. This is reflected by the width of the posteriors shown in Fig. 7.

The $\chi^{2}$ contributions to the Hillipop+VHL+lowTEB best fit of each of the very-high- $\ell$ datasets are $58 / 47$ (d.o.f.), $77 / 90$, and 651/710 for the SPT_low, SPT_high, and ACT datasets, respectively. None of these individual values indicate strong tension between the likelihood parts. We note that in all cases, the covariance matrices provided by the ACT and SPT groups, and used to compute the $\chi^{2}$, include non negligible, non-diagonal elements. For example, the visual impression from the SPT+low residuals, shown on Fig. C. 3 is not excellent, but the $\chi^{2}$ from this part of the fit given above has a PTE of $13 \%$, which is perfectly acceptable. 
F. Couchot et al.: Relieving tensions related to $A_{\mathrm{L}}$

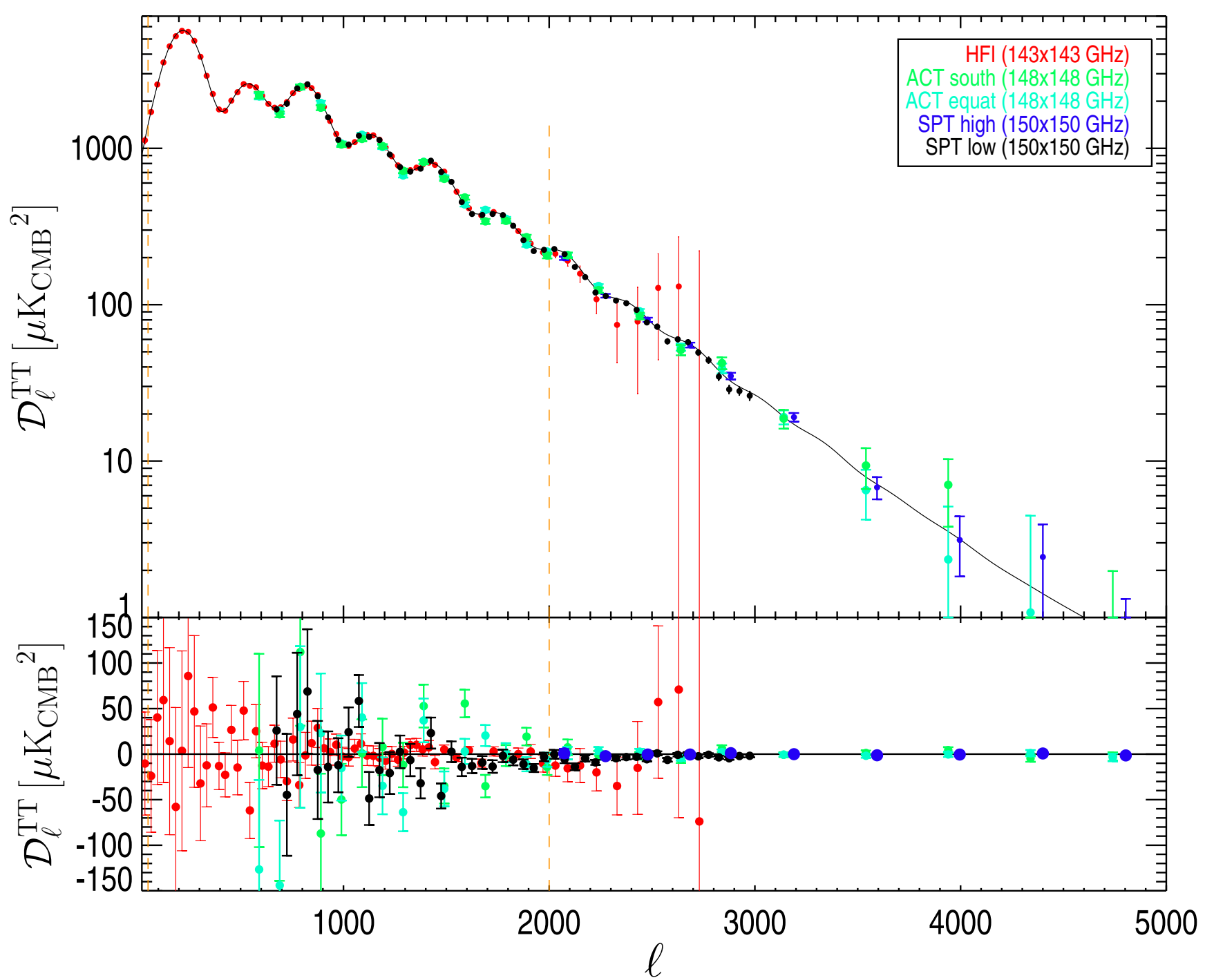

Fig. 6. Foreground-subtracted CMB cross-spectra of all the experiments used at $\simeq 150 \mathrm{GHz}$. The solid line is the best fit of the Hillipop+VHL combination and is subtracted to obtain the bottom residual plot. The Hillipop likelihood uses individual multipoles up to 2000 and window functions have been accounted for in the very-high- $\ell$ data.

\section{5. $A_{L}$ and $\tau$ results}

As a second step, we perform the same analysis as in Sects. 2 and 3.2, adding the VHL likelihood to Hillipop and consider the $A_{\mathrm{L}}$ profile-likelihood (with lowTEB) in Fig. 8. The result becomes

$A_{\mathrm{L}}=1.03 \pm 0.08 \quad($ Hillipop+lowTEB $+\mathrm{VHL})$,

now fully compatible with one.

As seen in Sect. 5, the inclusion of the VHL data does not greatly change the cosmological parameters but, as expected, strongly constrains all the foregrounds including, through correlations, the ones specific to Planck data (dust and point source amplitudes). This is shown in Fig. 9.

We note that all Hillipop nuisance amplitudes (but the point sources) represent coefficients scaling foreground templates: it is remarkable that, after adding the VHL likelihood, they all lie reasonably (at least those for which we have the sensitivity) around one, which is a strong support in favor of the coherence of the foregrounds description.
For $\tau$, combining the VHL with the Hillipop likelihood removes any sign of tension with lowTEB as shown in Fig. 10, and we obtain

$\tau=0.052 \pm 0.035 \quad$ (Hillipop + VHL)

which is in excellent agreement with the lowTEB measurement (Eq. (6)).

\subsection{Robustness of the results}

We tested a large number of configurations of the VHL dataset to establish whether the improvement comes from a particular one. Results are presented in Table 4.

It is difficult to draw firm conclusions from this exercise, since the number of extra nuisance parameters varyies in each case (see Table 3). The improvement on $A_{\mathrm{L}}$ is most significant when combining several datasets, but also satisfactory as soon as one combines at least two of them. We note that all these results are highly correlated with each other, sinc they all make use of Hillipop. Even though central values very close to 1.0 may 

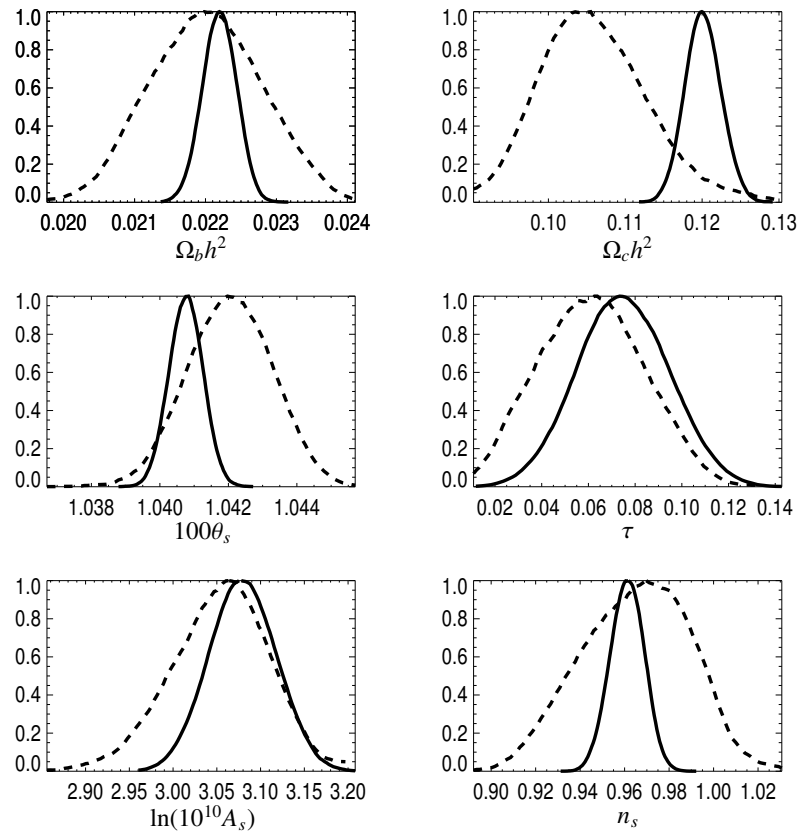

Fig. 7. Posterior distributions of the cosmological parameters obtained by sampling the Hillipop (soled line) and ACT +SPT (dashed) likelihoods independently. A prior of $\tau=0.07 \pm 0.02$ is used in both cases. For clarity, we only show the cosmological parameters, but all nuisances are sampled.

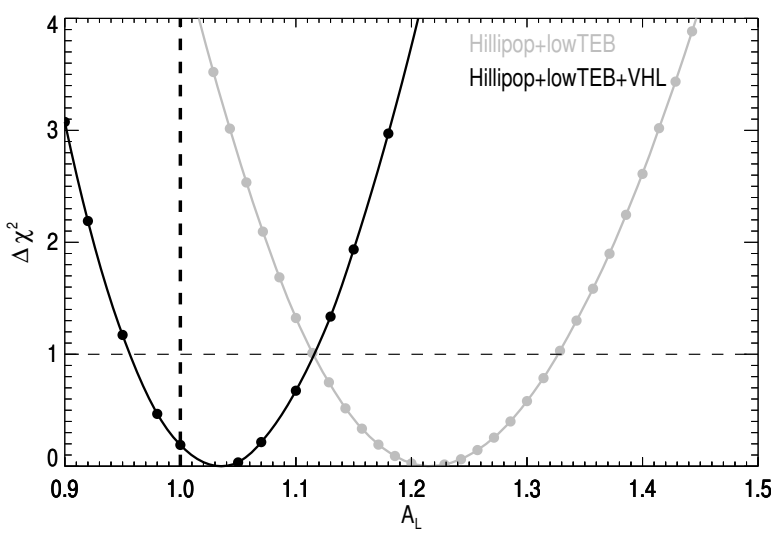

Fig. 8. Profile-likelihood for $A_{\mathrm{L}}$ reconstructed from the Hillipop+lowTEB likelihood (in grey) and adding the very-high- $\ell$ ACT and SPT data (VHL) discussed in the text (in black).

be preferable, it should be pointed out that there are several combinations that are compatible with 1.0 at the $\sim 1 \sigma$ level. This may indicate that the better the constraint on the high end of the power spectrum, the better the constraint on the $A_{\mathrm{L}}$ control parameter (the lensing effect on $C_{\ell}^{T T}$ is not only a smearing of peaks and troughs but also a redistribution of power towards the high $\ell$, above $\sim 3000$ ).

Since there is some overlap between SPT_low and SPT_high datasets, we expect some correlations between the SPT_low power-spectrum and, in particular, the $150 \mathrm{GHz}$ spectrum of SPT_high. To check the impact on the results, we either removed the entire $150 \mathrm{GHz}$ from SPT_high or the overlapping bins in multipole for each of the two datasets and re-ran the analysis. In all cases the results were similar with the combined one. For example, when removing the bins at $\ell<3000$ from the $150 \times 150 \mathrm{GHz}$ of SPT_high, we find $A_{\mathrm{L}}=0.99 \pm 0.08$, which is in good agreement with unity and with the results reported in Table 4. More checks are described in Appendix C.
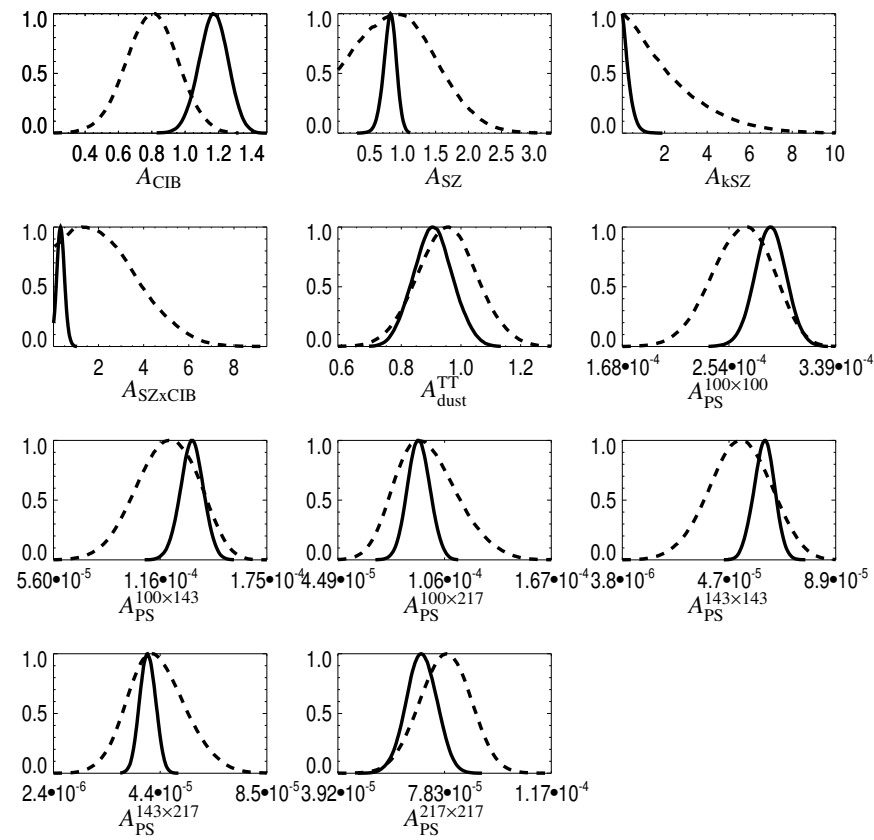

Fig. 9. Posterior distributions of the foreground Hillipop parameters with (solid line) and without (dashed line) the VHL likelihood. The definition of each parameter can be found in Table 1.

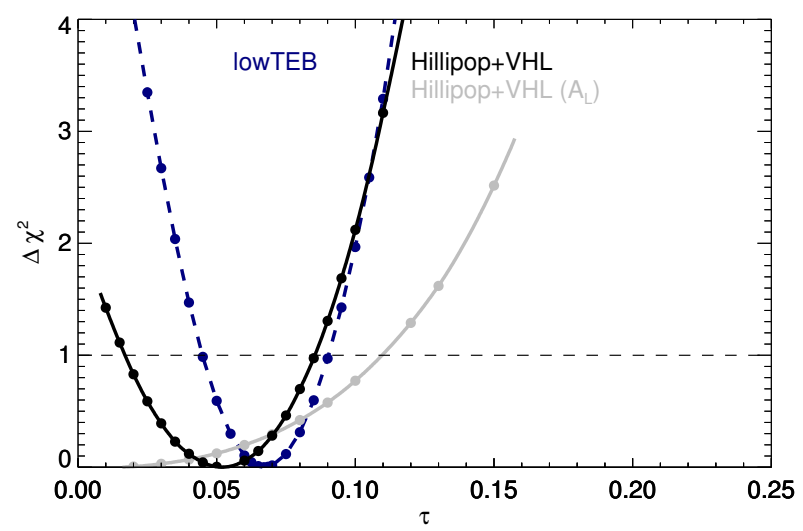

Fig. 10. Hillipop+VHL and lowTEB likelihood constraints on $\tau$. The grey profile shows the result for Hillipop+VHL when $A_{\mathrm{L}}$ is left free in the fits. The lowTEB one is in dashed blue.

Finally, we noticed that the SPT_high $220 \times 220$ spectrum lies slightly high with respect to the best fit (see Appendix C). Indeed, we get a better agreement in this case by increasing the contribution of the SPT dust amplitude. To check the impact on $A_{\mathrm{L}}$, we re-run the analysis, multiplying the dust level for all SPT spectra, $A_{\text {dust }}^{\mathrm{SPT}}$, by a factor of 3 and obtain

$A_{\mathrm{L}}=1.13 \pm 0.10\left(\mathrm{Hillipop}+\right.$ lowTEB $+\mathrm{SPT} \_$high, $\left.A_{\text {dust }}^{\mathrm{SPT}} \times 3\right)$
$A_{\mathrm{L}}=1.04 \pm 0.08\left(\mathrm{Hillipop}+\right.$ lowTEB $\left.+\mathrm{VHL}, A_{\text {dust }}^{\mathrm{SPT}} \times 3\right)$.

Compared with Table 4, the details of the SPT dust amplitude do not affect the final results.

\subsection{Where does the change on $A_{L}$ come from?}

Adding the ACT and SPT data lowered the $A_{\mathrm{L}}$ estimate. In this section, we try to pinpoint where the change came from.

As discussed in Sect. 2.1, Planck included the very-high- $\ell$ information in the Plik likelihood through a linear constraint 
Table 4. Results on $A_{\mathrm{L}}$ using Hillipop+lowTEB and various dataset combinations on the VHL side.

\begin{tabular}{|c|c|}
\hline Dataset & $A_{\mathrm{L}}$ \\
\hline None ................. & $1.22 \pm 0.11$ \\
\hline SPT_low ......... & $1.16 \pm 0.10$ \\
\hline SPT_high & $1.12 \pm 0.10$ \\
\hline $\mathrm{ACT} \ldots \ldots \ldots \ldots$ & $1.19 \pm 0.10$ \\
\hline SPT_low + SPT_high & $1.02 \pm 0.08$ \\
\hline SPT_low + ACT $\ldots$. & $1.09 \pm 0.09$ \\
\hline SPT_high + ACT & $1.12 \pm 0.09$ \\
\hline SPT_low + SPT_high + ACT $\ldots .$. & $1.03 \pm 0.08$ \\
\hline
\end{tabular}

Notes. For their exact definition see Sect. 4.

between the thermal and kinetic components of the SZ foreground. When combining Hillipop with the VHL likelihood, a similar correlation is observed that in our units reads:

$A^{\mathrm{kSZ}}+3.5 A^{\mathrm{tSZ}}=3.16 \pm 0.25$.

To check whether this correlation is sufficient to capture the essentials of the very-high- $\ell$ information, we re-run the profile analysis adding to Hillipop+lowTEB only the prior in Eq. (14) and measure:

$A_{\mathrm{L}}=1.26_{-0.10}^{+0.12} \quad$ (Hillipop+lowTEB+SZ-cor).

A comparison with Eq. (8) shows that, at least in our case, using this correlation does not capture the complexity of the full covariance matrix.

So, to check if the change came from a better constraint over all the foregrounds, we perform the following measurement: we use the Hillipop+lowTEB likelihood to determine $A_{\mathrm{L}}$ as in Sect. 3.2 but fixing all the nuisance parameters to the best-fit value of Hillipop+lowTEB+VHL likelihood. A profilelikelihood analysis gives

$A_{\mathrm{L}}=1.09 \pm 0.08$ (Hillipop+lowTEB, fixed nuisances),

compatible with unity at $\sim 1 \sigma$ as when using the very-high- $\ell$ data. We conclude that the shift for $A_{\mathrm{L}}$ seems to come from the better determination of the foregrounds parameters. To determine which particular foreground parameters impacts the $A_{\mathrm{L}}$ shift, we run an MCMC analysis sampling all the parameters (including $A_{\mathrm{L}}$ ) with the Hillipop+lowTEB and Hillipop+lowTEB+VHL likelihoods. The posterior distributions for $A_{\mathrm{L}}$ and the foregrounds parameters in common between Hillipop and VHL are shown on Fig. 11. It is difficult to single out a particular correlation and we conclude that the improvement comes from the overall better constraint of the foregrounds.

\section{Results on $\Lambda$ CDM parameters}

We have shown how the Hillipop likelihood is regularized by including the very-high- $\ell$ data. We have checked that it leads to results that are fully compatible with the lowTEB likelihood for $\tau$ and that their combination leads to an $A_{\mathrm{L}}$ value that is now compatible with one. We then combine the three likelihoods and fix $A_{\mathrm{L}}$ to one, to evaluate the impact on $\Lambda \mathrm{CDM}$ parameters. The comparison with the Planck published result is shown in Fig. 12. We note that:

- Plik and Hillipop likelihoods essentially share the same data;

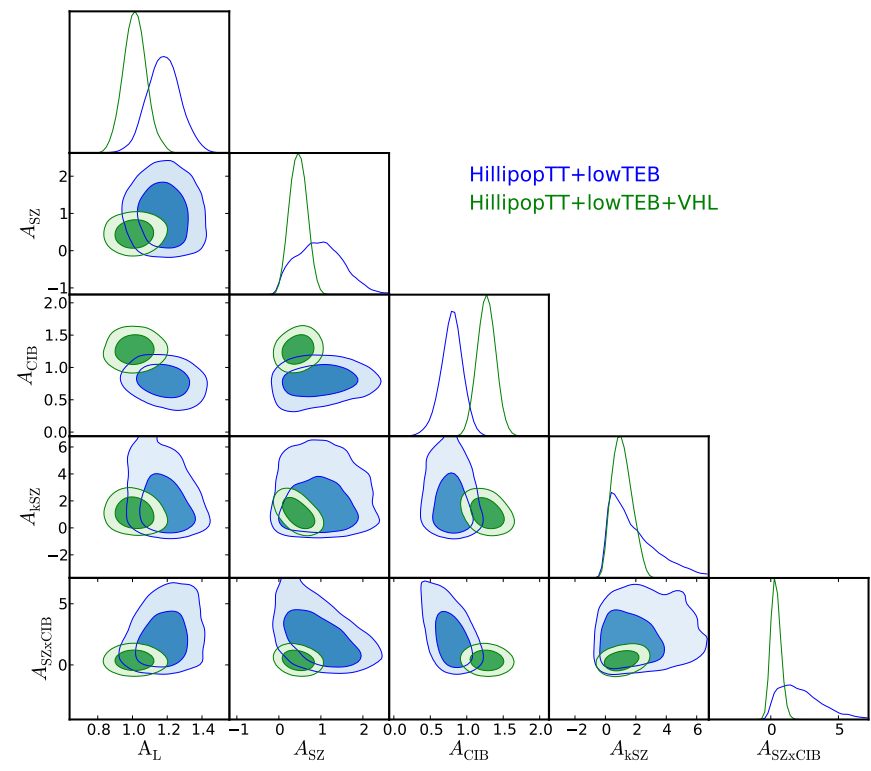

Fig. 11. Posterior distritions ( 68 and $95 \%$ levels) obtained from sampling the Hillipop+lowTEB and Hillipop+lowTEB+VHL likelihoods. We display the parameters most constrained by VHL.
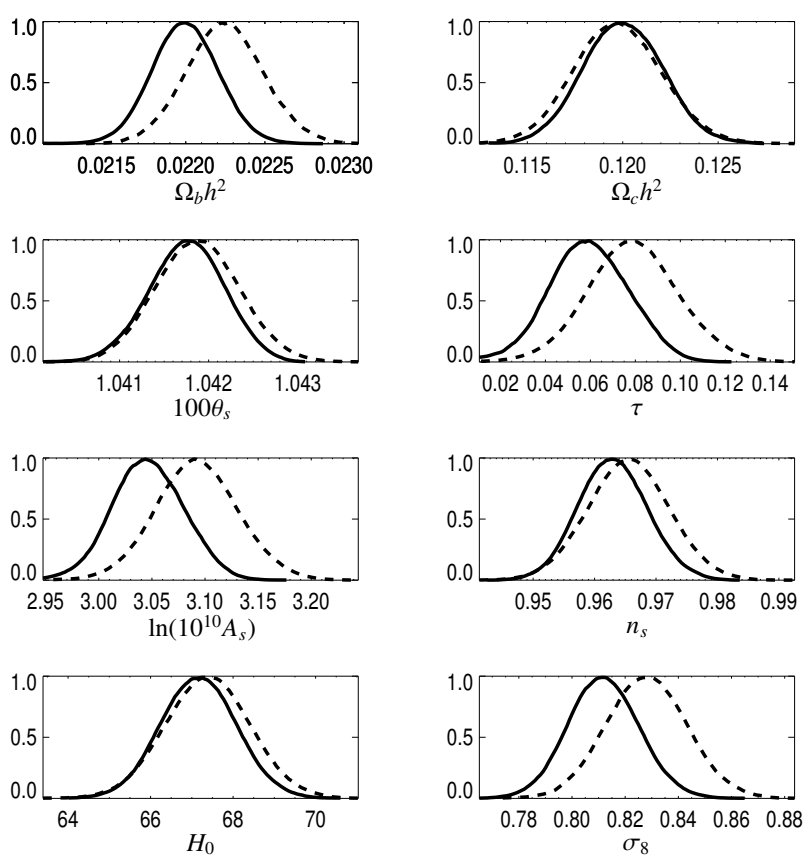

Fig. 12. Posterior distributions of the $\Lambda C D M$ cosmological parameters obtained with our regularized likelihood (Hillipop+lowTEB+VHL, full line), compared to the Planck baseline result (Plik+lowTEB, dashed line). We recall that this former also includes some VHL information through an SZ correlation discussed in PCP15. The last row shows derived parameters.

- the problem pointed out by $A_{\mathrm{L}}$ is not a second-order effect: it directly affects $\Omega_{\mathrm{b}}, \tau$, and $A_{\mathrm{s}}$ results;

- our regularized likelihood provides a lower $\sigma_{8}$ estimate.

These results are obtained using only CMB data. To check the overall consistency and constrain the parameters, we also make use of some robust extra-information by including recent baryon acoustic oscillations $(\mathrm{BAO})$ and supernovae $(\mathrm{SN})$ results. 
Table 5. Estimates of cosmological parameters using MCMC techniques for the six $\Lambda$ CDM parameters.

\begin{tabular}{lccc}
\hline \hline Parameter & Hillipop+lowTEB & $\begin{array}{c}\text { Hillipop+lowTEB } \\
\text { +VHL }\end{array}$ & $\begin{array}{c}\text { Hillipop+lowTEB } \\
+ \text { VHL+BAO+SN }\end{array}$ \\
\hline$\Omega_{\mathrm{b}} h^{2} \ldots \ldots \ldots$ & $0.02220 \pm 0.00023$ & $0.02203 \pm 0.00020$ & $0.02211 \pm 0.00018$ \\
$\Omega_{\mathrm{c}} h^{2} \ldots \ldots \ldots \ldots$ & $0.1193 \pm 0.0022$ & $0.1196 \pm 0.0020$ & $0.1183 \pm 0.0012$ \\
$100 \theta_{\mathrm{s}} \ldots \ldots \ldots$ & $1.04179 \pm 0.00043$ & $1.04181 \pm 0.00042$ & $1.04190 \pm 0.00038$ \\
$\tau \ldots \ldots \ldots$ & $0.071 \pm 0.019$ & $0.058 \pm 0.018$ & $0.062 \pm 0.017$ \\
$n_{\mathrm{s}} \ldots \ldots \ldots$ & $0.9644 \pm 0.0069$ & $0.9626 \pm 0.0055$ & $0.9654 \pm 0.0040$ \\
$\ln \left(10^{10} A_{\mathrm{s}}\right) \ldots \ldots$ & $3.068 \pm 0.037$ & $3.044 \pm 0.034$ & $3.048 \pm 0.033$ \\
\hline$H_{0} \ldots \ldots \ldots$ & $67.48 \pm 0.98$ & $67.21 \pm 0.91$ & $67.77 \pm 0.57$ \\
$\sigma_{8} \ldots \ldots \ldots$ & $0.816 \pm 0.015$ & $0.809 \pm 0.013$ & $0.807 \pm 0.013$ \\
\hline
\end{tabular}

Notes. First with our likelihood (Hillipop) and lowTEB. Then with our regularized likelihood (Hillipop+lowTEB+VHL, second column) and further adding some BAO and SN data (third column). Here $\theta_{\mathrm{s}}$, as computed by class, represents the exact angular size of the sound horizon and should not be identified with the CosmoMC $\theta_{\mathrm{MC}}$ parameter (see Appendix B).

The BAO are generated by acoustic waves in the primordial fluid, and can be measured today through the study of the correlation functions of galaxy surveys. Owing to the fact that their measurement is sensitive to different systematic errors than the $\mathrm{CMB}$, they help break the degeneracies, and are therefore further used in this paper to constrain the cosmological parameters. Here, we have used: the acoustic-scale distance ratio $D_{V}(z) / r_{\text {drag }}$ measurements ${ }^{6}$ from the $6 \mathrm{dF}$ Galaxy Survey at $z=0.1$ (Beutler et al. 2014), and from BOSS-LowZ at $z=0.32$. They have been combined with the BOSS-CMASS anisotropic measurements at $z=0.57$, considering both the line of sight and the transverse direction, as described in Anderson et al. (2014).

Type Ia supernovae had a major role in the discovery of late time acceleration of the Universe and constitutes a powerful cosmological probe complementary to CMB constraints. We have used the JLA compilation (Betoule et al. 2014), which covers a wide redshift range (from 0.01 to 1.2 ).

Table 5 gives the results obtained with Hillipop+lowTEB (i.e., using only Planck data), then adding ACT+SPT likelihoods (i.e., only CMB) and finally also adding the BAO and SNIa likelihoods. Using only Planck data, the Hillipop+lowTEB results (first column in Table 5) are almost identical to the ones reported for "Planck TT+lowP" in PCP15, but for $\tau$ and $A_{\mathrm{s}}$ that are smaller, as explained in Sects. 3.2 and 2.5.

As discussed throughout this paper, adding the very-high- $\ell$ data (second column) releases the tension on the optical depth, leading to a value of $\tau$ around 0.06 , as can be anticipated from Fig. 10. We also see a slight shift of $\Omega_{\mathrm{b}} h^{2}$ that is difficult to analyze since, at this level of precision, this parameter enters several areas of the Boltzmann computations (e.g., Hu \& White 1997). Then, adding the BAO and SN data (third column) increases the precision on the parameters but does not change substantially their value.

We note that in all cases, $\sigma_{8}$ is stable, e.g., Hillipop+lowTEB gives $\sigma_{8}=0.816 \pm 0.015$. This is only in mild tension with other astrophysical determinations such as weak lensing (Heymans et al. 2013) and Sunyaev-Zeldovich cluster number counts (Planck Collaboration XXIV 2016).

\footnotetext{
$6 D_{V}(z)$ is a function of the redshift $(z)$ and can be expressed in terms of the angular diameter distance and the Hubble parameter, $r_{\text {drag }}$, is the comoving sound horizon at the end of the baryon drag epoch.
}

\section{Conclusion}

In this work, we have investigated the deviation of the $A_{\mathrm{L}}$ parameter from unity and have found it to be of $2.6 \sigma$, using the Plik high- $\ell$ and lowTEB low- $\ell$ Planck likelihoods. For these demanding tests, we chose to consistently use the profile-likelihood method, which is well-suited to such studies. We first showed how this $A_{\mathrm{L}}$ deviation is related to a difference in the $\tau$ estimations when performed using Plik or lowTEB alone.

The Hillipop likelihood has been built based on different foreground and nuisance parametrization. We have shown that Hillipop alone only very loosely constrains $A_{\mathrm{L}}$ towards high values, and that its $\tau$ estimate lies closer to the low- $\ell$ measurement, albeit on the high-end side.

We then added to Hillipop, high angular resolution CMB data from the ground-based ACT and SPT experiments to further constrain the high- $\ell$ part of the CMB power spectrum and the foreground parameters. Cosmological parameters derived from this setup are shown to be more self-consistent, in particular the reconstructed $\tau$ value is coherent with the low- $\ell$ determination that was extracted from the lowTEB likelihood. They also pass the $A_{\mathrm{L}}=1.0$ test.

We have shown that this regularization is quite robust against the details of the very-high- $\ell$ datasets used, and specific foreground hypotheses. We have also shown that it is not only related to a better determination of the foreground amplitudes but also seems to lie in their correlations.

The cosmological parameters determined from this combined CMB likelihood are also stable when adding BAO and SNIa likelihoods. This is, in particular, the case for $\sigma_{8}$ that we always find close to 0.81 . With respect to the cosmological parameters derived by the Planck Collaboration, the main differences concern $\tau$ and $A_{\mathrm{s}}$, to which the former is directly correlated, and $\Omega_{\mathrm{b}} h^{2}$, which shifts by a fraction of $\sigma$. Other parameters are almost identical.

Improving on $A_{\mathrm{L}}$ is a delicate task and it seems that the source of the regularization cannot be easily pinpointed. The choices of the Hillipop likelihood impact on the correlations between all the parameters, yielding a $\tau$ estimate in smaller tension with the low- $\ell$ likelihood. But this was not sufficient to relieve the $A_{\mathrm{L}}$ tension. It is only by further constraining foregrounds using very-high- $\ell$ likelihoods that we were able to obtain a coherent picture over a broad range of multipoles. 
F. Couchot et al.: Relieving tensions related to $A_{\mathrm{L}}$

One cannot exclude that the $A_{\mathrm{L}}$ deviation from unity still partly results from an incomplete accounting of some residual systematics in the ACT, SPT, or Planck data.

During the review of this article, the Planck Collaboration released an estimate of the optical depth based on HFI cleaned maps (Planck Collaboration Int. XLVII 2016) and using the Lollipop likelihood (Mangilli et al. 2015). This new low- $\ell$-only result, $\tau=0.058 \pm 0.012$, increases the tension with the high- $\ell$ likelihoods to $2.6 \sigma$ with Plik and $1.5 \sigma$ with Hillipop and is fully compatible with our Hillipop+VHL combination (Eq. (13)).

Acknowledgements. We thank Guilaine Lagache for building and providing the point-source masks cleaned from Galactic compact structures, Marian Douspis for providing the SZ templates, and Marc Betoule for the development of the $C$ version of the JLA likelihood.

\section{References}

Anderson, L., Aubourg, É., Bailey, S., et al. 2014, MNRAS, 441, 24 Béthermin, M., Daddi, E., Magdis, G., et al. 2012, ApJ, 757, L23

Betoule, M., Kessler, R., Guy, J., et al. 2014, A\&A, 568, A22 Beutler, F., Saito, S., Brownstein, J. R., et al. 2014, MNRAS, 444, 3501 Blanchard, A., \& Schneider, J. 1987, A\&A, 184, 1

Blas, D., Lesgourgues, J., \& Tram, T. 2011, J. Cosmol. Astropart. Phys., 7, 034 Calabrese, E., Slosar, A., Melchiorri, A., Smoot, G. F., \& Zahn, O. 2008, Phys. Rev. D, 77, 123531
Das, S., Louis, T., Nolta, M. R., et al. 2014, J. Cosmol. Astropart. Phys., 4, 14 Di Valentino, E., Melchiorri, A., \& Silk, J. 2016, Phys. Rev. D, 93, 023513 Dunkley, J., Calabrese, E., Sievers, J., et al. 2013, J. Cosmol. Astropart. Phys., 7, 25

George, E. M., Reichardt, C. L., Aird, K. A., et al. 2015, ApJ, 799, 177 Heymans, C., Grocutt, E., Heavens, A., et al. 2013, MNRAS, 432, 2433 Hu, B., \& Raveri, M. 2015, Phys. Rev. D, 91, 123515

Hu, W., \& White, M. 1997, ApJ, 479, 568

James, F. 2007, Statistical Methods in Experimental Physics (World Scientific) Lesgourgues, J. 2011, ArXiv e-prints [arXiv: 1104 . 2934]

Lewis, A., \& Challinor, A. 2006, Phys. Rep., 429, 1

Lewis, A., Challinor, A., \& Lasenby, A. 2000, ApJ, 538, 473

Mangilli, A., Plaszczynski, S., \& Tristram, M. 2015, MNRAS, 453, 3174

Planck Collaboration IX. 2014, A\&A, 571, A9

Planck Collaboration XVI. 2014, A\&A, 571, A16

Planck Collaboration XXX. 2014, A\&A, 571, A30

Planck Collaboration I. 2016, A\&A, 594, A1

Planck Collaboration VIII. 2016, A\&A, 594, A8

Planck Collaboration XI. 2016, A\&A, 594, A11

Planck Collaboration XIII. 2016, A\&A, 594, A13

Planck Collaboration XXII. 2016, A\&A, 594, A22

Planck Collaboration XXIV. 2016, A\&A, 594, A24

Planck Collaboration Int. XVI. 2014, A\&A, 566, A54

Planck Collaboration Int. XXX. 2016, A\&A, 586, A133

Planck Collaboration Int. XLVII. 2016, A\&A, 596, A108

Porter, F. 1996, Nucl. Instrum. Meth. Phys. Res. A, 368, 793

Reichardt, C. L., Shaw, L., Zahn, O., et al. 2012, ApJ, 755, 70

Story, K. T., Reichardt, C. L., Hou, Z., et al. 2013, ApJ, 779, 86

Tristram, M., Macías-Pérez, J. F., Renault, C., \& Santos, D. 2005, MNRAS, 358, 833 


\section{Appendix A: Class vs. camb results}

Our results were obtained using the class v2.3.2 Boltzmann code, which computes the temperature and polarization power spectra by evolving the cosmological background and perturbation equations. Besides being very clearly written in $C$ and modular, class has a very complete set of precision parameters in a single place that enables us to study them efficiently. This makes it a perfect tool for developing modern high-precision cosmological projects.

We revisit the agreement between class and camb at the level required to estimate $A_{\mathrm{L}}$. For this purpose, we fix the cosmology to the Planck TT+lowP best fit and run class with three different settings:

- the class default ones;

- the high-quality (HQ) ones that are being used to obtain some smooth profile-likelihoods and are given in Table B.1;

- the very high-quality (VHQ) ones corresponding to the maximal precision one can reasonably reach on a cluster ${ }^{7}$ and that are provided in class in the file cl_ref.pre.

For camb, we use the corresponding spectrum released in the Planck Legacy Archive. We compare the spectra on Fig. A.1 Generally speaking the agreement is at the $(\mu \mathrm{K})^{2}$ level, which is below one percent agreement over the whole range. In practice, this means it does not impact on the $\Lambda$ CDM parameters, as discussed in Like15.

The low- $\ell$ difference is not very important for data analysis since experiments are limited there by the cosmic variance. The asymptotic $\simeq 1(\mu \mathrm{K})^{2}$ offset is more worrying and some $\Lambda$ CDM extensions could be sensitive to this difference. Since the class spectrum is slightly lower than the camb one precisely in the high- $\ell$ region where $A_{\mathrm{L}}$ has the largest impact, it should lead to a higher $A_{\mathrm{L}}$ value. This is indeed observed and discussed in Sect. 2.4, but has a small effect on $A_{\mathrm{L}}$.

Finally we note that all class settings give similar results, in particular in their high $\ell$ region, and that our HQ settings (used for profile-likelihoods) are smoother than the default ones and similar to the most extreme ones (VHQ).

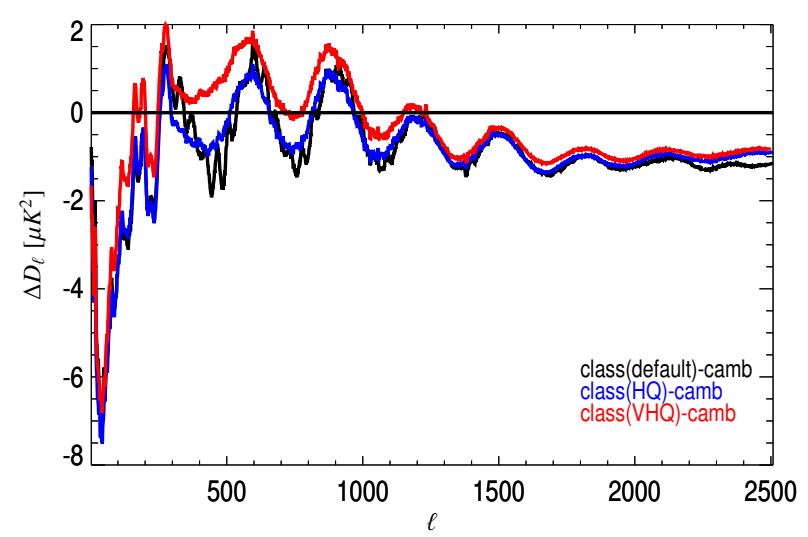

Fig. A.1. Difference of $D_{\ell} \equiv \ell(\ell+1) C_{\ell}^{T T} / 2 \pi$ power spectra between class and camb for the same fiducial cosmology. For class several precision settings are tested: the default ones (in black), the high-quality (in blue) and very high-quality ones (in red) as described in the text.

\footnotetext{
7 This single shot run last several minutes on 8 cores and requires about 30GB of memory.
}

Table B.1. High-precision settings of the class non-default parameters used in the profile-likelihood constructions.

\begin{tabular}{lc}
\hline \hline \multicolumn{1}{c}{ class parameter } & Value \\
\hline tol_background_integration & $10^{-3}$ \\
tol_thermo_integration & $10^{-3}$ \\
tol_perturb_integration & $10^{-6}$ \\
reionization_optical_depth_tol & $10^{-5}$ \\
l_logstep & 1.08 \\
l_linstep & 25 \\
perturb_sampling_stepsize & 0.04 \\
delta_1_max & 1000 \\
accurate_lensing & 1
\end{tabular}

Notes. The last parameter (accurate_lensing) is only useful (and used) to model in details the very-high- $\ell$ tail (typically above 4000) and is only used with our ACT and SPT likelihoods.

\section{Appendix B: Optimization of profile-likelihoods}

The computation of genuine profile-likelihoods requires exquisite minimization precision. Since the $68 \%$ confidence intervals are obtained by thresholding the profiles at 1 , we need a precision on $\chi_{\min }^{2}$, well below 0.1 , which is challenging for a numerical method without analytic gradients. It has already been shown in Planck Collaboration Int. XVI (2014) how using the Minuit package and increasing the class precision parameters to high values (still keeping a reasonable computation time) enables us to achieve this goal.

Besides revisiting our high-precision parameters that are shown in Table B.1, we have improved our strategy further by using the following scheme:

1. we use the pico software ${ }^{8}$ to pre-compute a best fit solution. pico is based on the interpolation between spectra trained on the camb Boltzmann solver. It is very fast and the minimization converges rapidly owing to the smoothness between models:

2. since camb computes only an approximation of the angular size of the sound horizon $\left(\theta_{\mathrm{MC}}\right)$ while class computes it exactly $\left(\theta_{\mathrm{s}}\right)$ we perform the change of variables by fixing the previous best-fit estimates and performing a $1 \mathrm{D}$ fit to $\theta_{\mathrm{s}}$ only;

3. we then have a good starting point to class and perform the minimization with a high-precision strategy (Migrad, strategy $=2$ level);

4. optionally: in some rare cases, the estimated minimum is not accurate enough (this can be identified by checking the profilelikelihood continuity). In this case, we use several random initialization points (typically 10) near the previous minimum, perform the same minimization, and keep the lowest $\chi_{\min }^{2}$ solution.

In all cases we have checked that the pico results are similar to the final class ones but consider the latter to give more precise results since pico implements only an approximation to the $A_{\mathrm{L}}$ parameter and was trained on an old camb version.

\footnotetext{
8 https://sites.google.com/a/ucdavis.edu/pico
} 


\section{Appendix C: Very-high- $\ell$ consistency}

Here we provide more details on the checks we performed to assess the ACT, SPT, and Planck spectra compatibility in the very-high- $\ell$ regime. Figures C.1-C.3 show the detailed fit of each component for each dataset. The components are obtained from the templates scaled according to the combined Hillipop+lowTEB+VHL fitted cosmological and nuisance parameters. The general agreement on the very-high- $\ell$ side is very good as shown by the $\chi^{2}$ for each dataset given in Sect. 4.4. We note that the points are largely correlated and that the exact $\chi^{2}$ computation involves the full covariance matrices provided by each experiment, which all contain non-negligible off-diagonal terms. For example, the $\chi^{2}$ from the SPT_low part is 58 for $47 \mathrm{deg}$ of freedom, which has a $13 \%$ PTE. Some small excess in data seems to show up in the SPT_high $220 \times 220 \mathrm{GHz}$ spectrum above $\ell=3000$. In Sect. 4.6 we checked that this does not influence any of our results.

To check that the overlap in the data used on the SPT_lowand SPT_high datasets, we did three additional profile likelihood analyses for $A_{\mathrm{L}}$. First, we ignored the $150 \mathrm{GHz}$ data from
Table C.1. Results on $A_{\mathrm{L}}$ using Hillipop+lowTEB and VHL, making various attempts at removing the correlated part between SPT_high and SPT_low: ${ }^{(a)}$ w/o $150 \mathrm{GHz}$ data $;{ }^{(b)}$ w/o overlapping bins; ${ }^{(c)}$ w/o overlapping bins.

\begin{tabular}{|c|c|}
\hline Dataset & $A_{\mathrm{L}}$ \\
\hline SPT_low + SPT_high ${ }^{a}+\mathrm{ACT}$ & $1.00 \pm 0.08$ \\
\hline SPT_low ${ }^{b}+$ SPT_high + ACT & $1.05 \pm 0.08$ \\
\hline SPT_low + SPT_high ${ }^{c}+\mathrm{ACT}$ & $0.99 \pm 0.08$ \\
\hline
\end{tabular}

SPT_high, recomputing accordingly the inverse covariance matrix used in the $\chi^{2}$ computation. We also tested the impact of removing in the SPT_low spectrum all bins above $\ell=3000$ (which are accounted for in the SPT_high part. To do this, we again recomputed the inverse covariance matrix. Finally, we omit in the SPT_high part bins at $150 \mathrm{GHz}$ at $\ell<3000$. These results are reported in Table C.1. All are compatible with each other and with unity. 


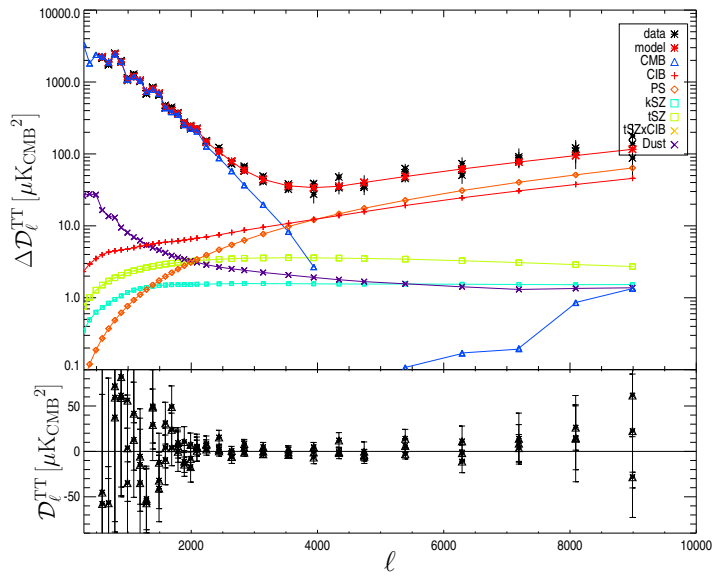

(a) ACT_equat $148 \times 148 \mathrm{GHz}$

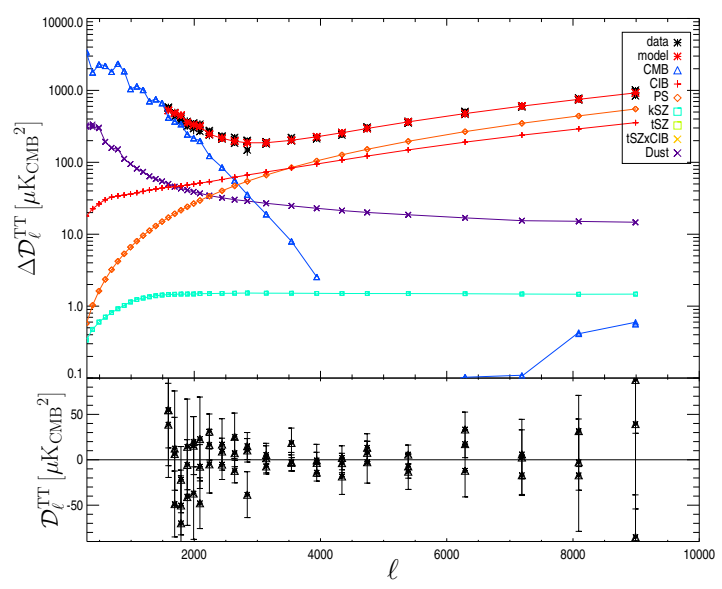

(c) ACT_equat $218 \times 218 \mathrm{GHz}$

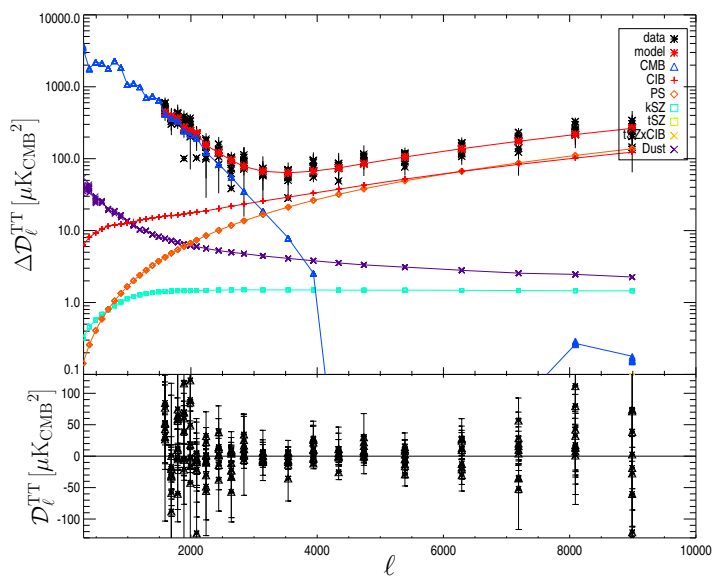

(e) ACT_south $148 \times 218 \mathrm{GHz}$

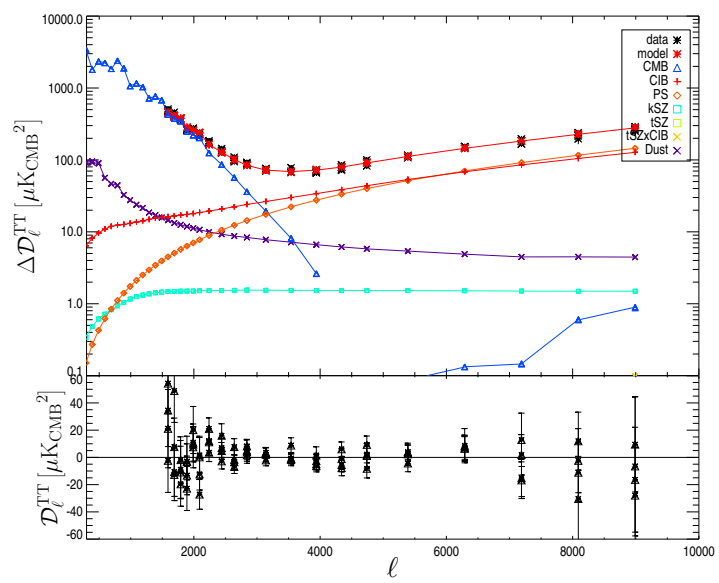

(b) ACT_equat $148 \times 218 \mathrm{GHz}$

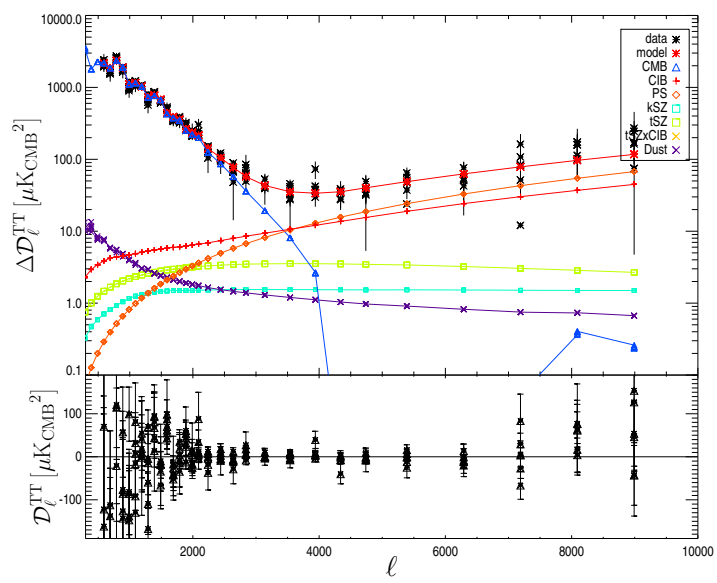

(d) ACT_south $148 \times 148 \mathrm{GHz}$

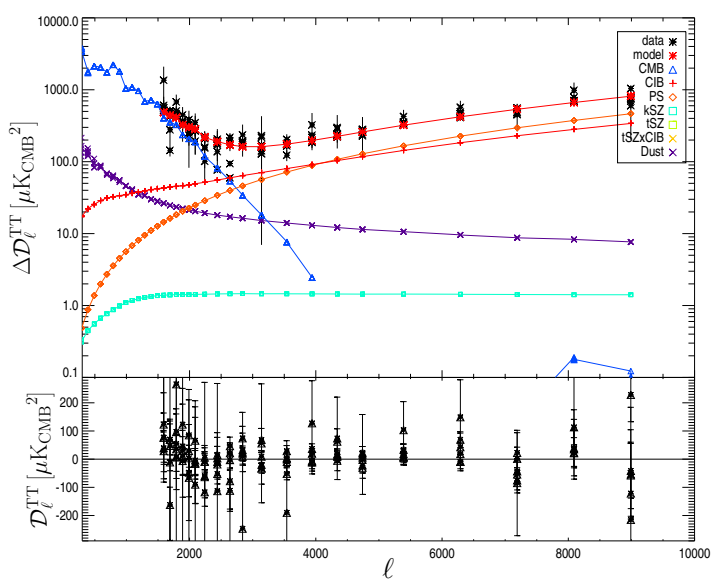

(f) ACT_south $218 \times 218 \mathrm{GHz}$

Fig. C.1. Power spectra of CMB and foregrounds as fitted by the Hillipop+VHL likelihood, compared with ACT data. The use of window functions explain why the CMB component sometimes reappears at the very end of the multipole range. 
F. Couchot et al.: Relieving tensions related to $A_{\mathrm{L}}$

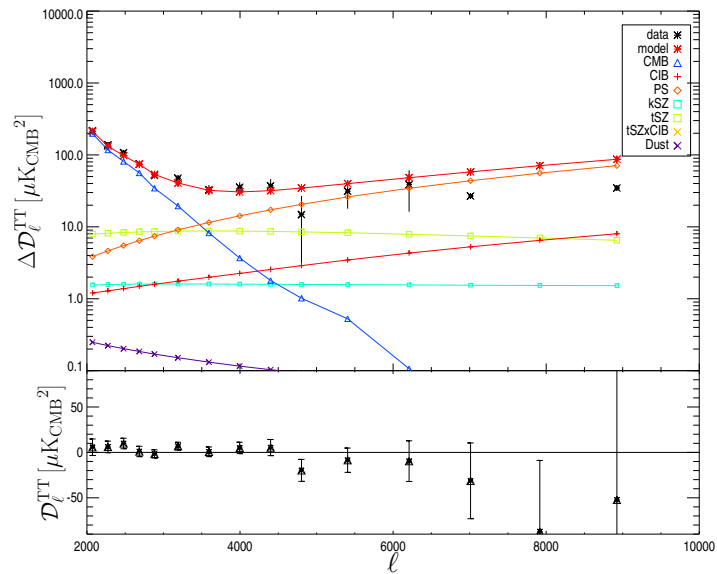

(a) SPT_high $95 \times 95 \mathrm{GHz}$

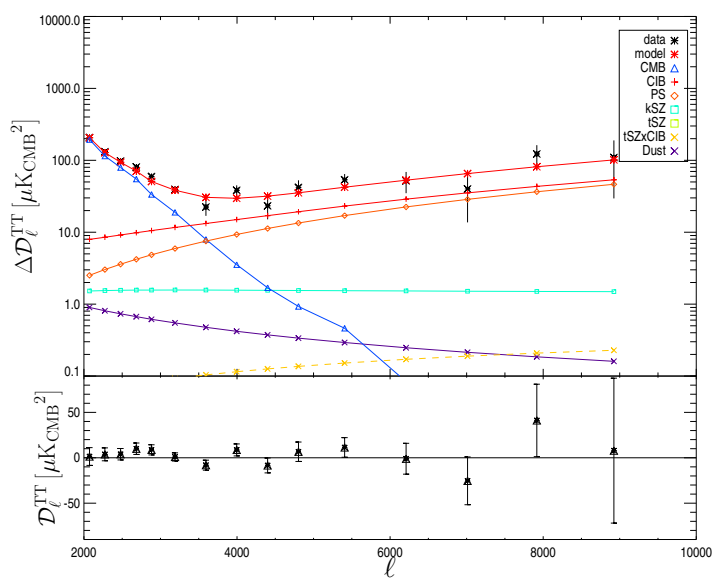

(c) SPT_high $95 \times 220 \mathrm{GHz}$

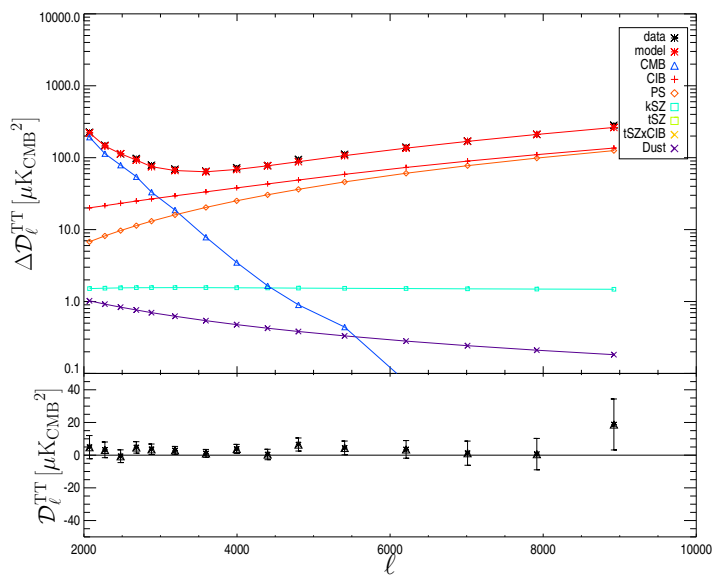

(e) SPT_high $150 \times 220 \mathrm{GHz}$

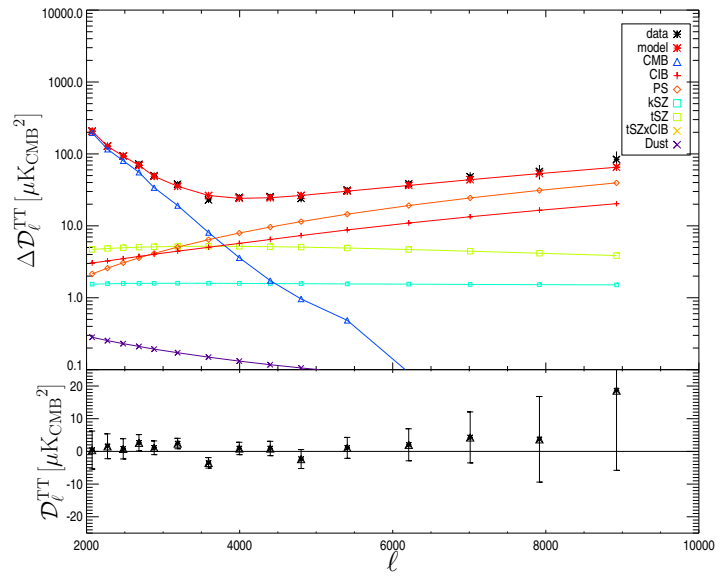

(b) SPT_high $95 \times 150 \mathrm{GHz}$

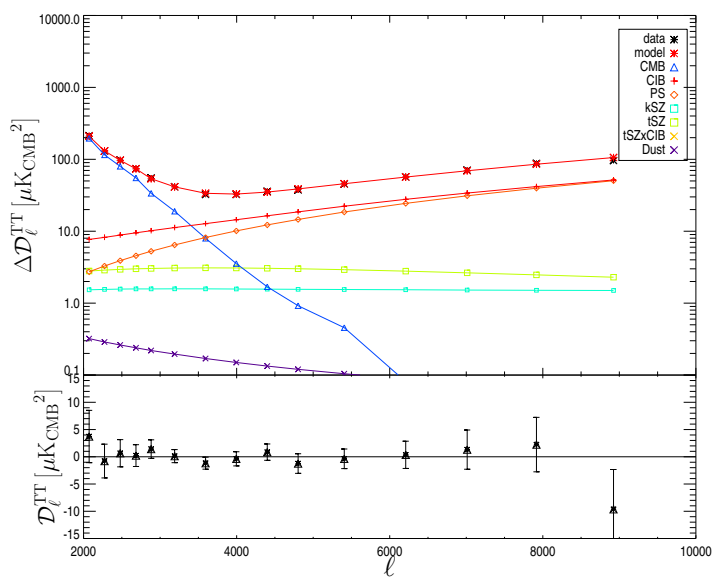

(d) SPT_high $150 \times 150 \mathrm{GHz}$

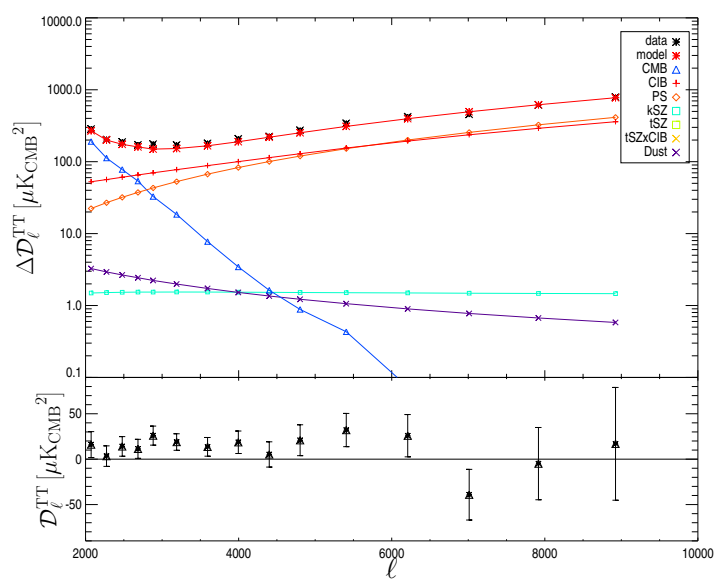

(f) SPT_high $220 \times 220 \mathrm{GHz}$

Fig. C.2. Power spectra of CMB and foregrounds as fitted by the Hillipop+VHL likelihood compared with SPT_high data. 
A\&A 597, A126 (2017)

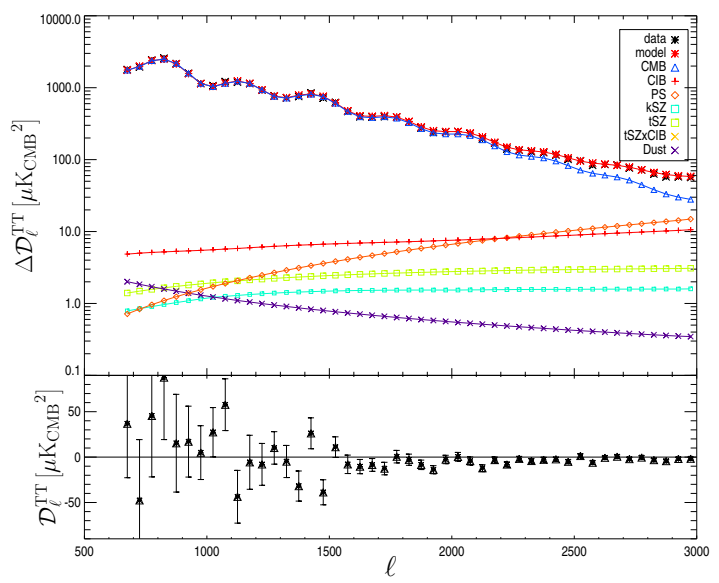

(a) SPT_low $150 \times 150 \mathrm{GHz}$

Fig. C.3. Power spectra of CMB and foregrounds as fitted by the Hillipop+VHL likelihood compared with SPT_low data. 